\title{
Interference and Coverage Analysis for Terahertz Networks with Indoor Blockage Effects and Line-of-Sight Access Point Association
}

\author{
Yongzhi Wu, Student Member, IEEE, Joonas Kokkoniemi, Member, IEEE, Chong Han, Member, IEEE \\ and Markku Juntti, Fellow, IEEE
}

\begin{abstract}
Providing high-bandwidth and fast-speed links, wireless local area networks (WLANs) in the Terahertz (THz) band have huge potential for various bandwidth-intensive indoor applications. However, due to the specific phenomena in the THz band, including severe reflection loss, indoor blockage effects, multi-path fading, the analysis on the interference and coverage probability at a downlink is challenging. In this paper, indoor blockage effects caused by the walls and human bodies are analyzed. Next, a statistical $\mathrm{THz}$ channel model is proposed to characterize the $\mathrm{THz}$ indoor propagation. In light of these, the moment generating functions of the aggregated interference and theoretical expressions for the mean interference power are derived. As a result, the approximated coverage probability and average network throughput are derived. Extensive numerical results show that for the nearest access point (nearest-AP) user association scheme, the optimal AP density is $0.15 / \mathrm{m}^{2}$, which results in the coverage probability reaches $93 \%$ and the average network throughput is $30 \mathrm{Gbps} / \mathrm{m}^{2}$. In addition, by adopting a novel line-of-sight access point (LoS-AP) user association mechanism, the coverage probability and the average network throughput can be further improved by 3 percent and $2 \mathrm{Gbps} / \mathrm{m}^{2}$, respectively.
\end{abstract}

Index Terms-Terahertz communications, Wireless local area networks, Stochastic geometry, Interference, Coverage probability.

\section{INTRODUCTION}

$\mathbf{R}$ ECENTLY, increasing demand for broader bandwidths has motivated the adoption of the Terahertz $(\mathrm{THz})$ band (0.1-10 THz). The THz communication has a huge potential to employ an ultra-wide bandwidth, which has been envisioned as a key technology for the sixth-generation (6G) wireless systems and beyond [2]. In order to facilitate the development of $\mathrm{THz}$ communications, the Federal Communication Commission has recently opened up a new category of experimental licenses for $6 \mathrm{G}$ communication systems or beyond between 95 $\mathrm{GHz}$ and $3 \mathrm{THz}[3]$.

This paper was presented in part at the IEEE Globecom workshop, HI, US, December 2019 [1]. Chong Han acknowledges the in-part support by Ministry of Education of China Joint Research Fund under Grant No. 6141A02033350, and National Natural Science Foundation of China (NSFC) under Grant No. 61701300. Yongzhi Wu and Chong Han are with Terahertz Wireless Communications (TWC) Laboratory, Shanghai Jiao Tong University, Shanghai, China (Email: \{yongzhi.wu, chong.han\}@sjtu.edu.cn).

Joonas Kokkoniemi and Markku Juntti acknowledge the support by Horizon 2020, European Union's Framework Programme for Research and Innovation under grant agreement no. 871464 (ARIADNE), and in part by the Academy of Finland 6Genesis Flagship under grant no. 318927. They are with Centre for Wireless Communications, University of Oulu, Oulu, Finland (Email: \{Joonas.Kokkoniemi, markku.juntti\}@oulu.fi).
With the great advantage of spectrum resources, $\mathrm{THz}$ communications is expected to improve the network capacity of wireless networks, such as the next-generation wireless local area networks (WLANs), which enables ultra-fast links between access points (APs) and user equipment (UE). The Terahertz wireless local area networks (Tera-WLANs) can realize high connectivity density, as more than 10 billion wireless devices, e.g., mobile phones and laptops, will be connected in the near future [4]. In addition, the bandwidthintensive applications across static and mobile users, such as virtual/augmented reality (VR/AR), 3D holographic calls, among other diverse low-latency applications, can come to reality in Tera-WLANs [1], [5].

Despite the wonderful vision and the great promise of $\mathrm{THz}$ networks, when it comes to the $\mathrm{THz}$ band, stringent challenges are encountered as a result of the distinctive features of $\mathrm{THz}$ wave propagation. First, compared to the lower frequency bands, including the ultra-high-frequency (UHF) and millimeter wave (mmWave) electromagnetic (EM) waves, the $\mathrm{THz}$ band experiences much higher free space path loss as it increases quadratically with frequency. Even one-meter communication distance at $0.67 \mathrm{THz}$ can lead to a free space path loss in the excess of $90 \mathrm{~dB}$. Beamforming and massive multiple input multiple output (MIMO) enable directional antennas with high antenna gains to compensate for the severe path loss [6]. Second, the atmospheric effects may attenuate the $\mathrm{THz}$ wave propagation and cause the molecular absorption loss, particularly obvious at some $\mathrm{THz}$ frequencies, which is not observed at lower frequency bands [7]. Due to the existence of the absorption peaks, the $\mathrm{THz}$ band is divided into $\mathrm{THz}$ windows with different widths, which are also dependent on the transmission distance. Third, reflection and scattering losses in the $\mathrm{THz}$ band become stronger [8], which results in the decrease of the number of the dominant rays, since the signal power of $\mathrm{THz}$ wave becomes very weak when it is reflected or scattered two or more times. Thus, the smallscale fading model caused by the multi-path propagation, such as Rayleigh fading, may not be applicable in the $\mathrm{THz}$ band [9]. Furthermore, $\mathrm{THz}$ waves nearly lose the ability to go through the blockages due to the very high penetration loss. For instance, $45.6 \mathrm{~dB}$ of attenuation is observed at 0.31 $\mathrm{THz}$ when the transmission is obstructed by a human hand [10]. Hence, the average coverage distance and the resulting cell size of a base station or an access point need to be redefined, especially in an indoor environment encountering 
various blockage effects.

As a promising pillar technology for the coming of $6 \mathrm{G}$ era, the Tera-WLANs need to be well designed in terms of the coverage probability, which is a widely used performance metric to quantify the reliability of wireless communications and has a strong impact on the quality of service [11]. While the highly directional antennas are used, the interference still limits significantly the coverage of the $\mathrm{THz}$ networks. In this paper, we first propose a system framework for the indoor Tera-WLANs by modeling the interference region with wall blockage effects, and derive the line-of-sight (LoS) and non-line-of-sight (NLoS) propagation probabilities caused by human blockage. We further develop a statistical indoor $\mathrm{THz}$ channel model by approximating the multi-ray $\mathrm{THz}$ channel. In light of this, we derive the moment generating functions (MGFs) of the aggregated interference from the interfering APs at the UE with directional antenna radiation and the mean interference power. Finally, we derive the coverage probability and the average network throughput for the Tera-WLANs.

The contributions of this work are summarized as follows:

- We propose a system framework for the indoor TeraWLANs and demonstrate that the THz APs can be classified into LoS, NLoS and non-interfering, caused by blockage effects. In particular, we model the wall obstruction as Manhattan Poisson line processes and the human blockage as random cylinder processes.

- We develop a statistical indoor THz channel model, which has the approximately identical fading distribution with the multi-ray $\mathrm{THz}$ channel model, and characterizes the large-scale fading and the small-scale fading that are well validated by numerical results.

- We derive the mean interference power in the presence of the indoor blockage effects, the multi-path fading and directional antenna radiation for three-dimensional (3D) Tera-WLANs. Furthermore, we derive the analytical expressions of the coverage probability and the average network throughput. We obtain a closed-form expression of the coverage probability to calculate the optimal AP density.

- We propose two user association schemes, namely, nearest-AP user association and LoS-AP user association, for the indoor Tera-WLANs. Through extensive numerical evaluations, we demonstrate that the LoSAP user association is better than the classic nearestAP scheme, by improving the coverage probability and average network throughput.

The rest of this paper is organized as follows. Related work is surveyed in Section II. The network model with indoor blockage effects is described in Section III. Section IV presents the statistical channel model and antenna pattern. The interference analysis is detailed in Section V. Then Section VI derives the network coverage probability. Numerical results for performance evaluation are elaborated in SectionVII. Finally, the paper is concluded in Section VIII.

\section{RELATED WORK}

The method of stochastic geometry has been widely used in analyzing the system performance in wireless networks. The most popular idea of stochastic geometry is to model the base stations as Poisson point processes [12], [13]. An important and popular assumption is the Rayleigh fading on the desired link, with which the exact distribution for the signal-tointerference-plus-noise ratio (SINR) could be obtained [14]. Tractable analysis for coverage and achievable rates can be offered for a fixed user by considering base stations are randomly distributed around it [15]. However, a major downside is that a closed-form expression for the probability density function (PDF) of the aggregated interference does not exist [14].

When moving to higher frequencies, such as mmWave and $\mathrm{THz}$ bands, directional antenna techniques are used to compensate for the high path loss, which introduces random antenna gains for the interfering signals. Different antenna radiation patterns are proposed in [16]-[20]. With a directional beam, the impact of the beam alignment on the coverage is taken into account in [21], [22]. For most of these studies, the antenna radiation pattern is considered in two-dimensional (2D) networks, which however are not suitable in more realistic 3D networks [9], [23].

Due to the strong penetration loss, the blockage effect of the randomly located buildings is analyzed in the outdoor mmWave cellular networks [16] and the cylinder blockage such as the human body has been widely studied in the mmWave networks [17], [19], [24], [25]. In a stochastic geometry framework, some approaches are developed to improve the coverage of outdoor networks, where the signal outage may occur due to the shadowing and blockage, including the base station co-operation [26] and relay aided transmission [27]. In an indoor environment, which has far more complex propagation conditions than outdoor environments [28], another typical blockage, the wall blocker, has not been investigated or modeled as a short finite line with random orientation in most of the existing studies. Besides the large-scale channel gain, the small-scale fading in the system model is considered as different distributions, such as Nakagami-m fading [16] and a log-normal distribution [27]. Nevertheless, such generic fading models have not been compared with real channel measurements and are not derived by the theoretical analysis, which may therefore result in an inaccurate estimation of the actual channel behaviors [20].

Focusing on $\mathrm{THz}$ communications, in addition to the free space path loss and absorption loss, the reflection or scattering loss in the $\mathrm{THz}$ band is higher than the lower frequencies, which causes the decrease of the number of dominant rays. Therefore, the existing fading models in the microwave and mmWave bands are not applicable in the $\mathrm{THz}$ band [9] and a new statistical model for the multi-path fading is needed. The studies in [29], [30] develop a statistical channel model in the $\mathrm{THz}$ band, by extracting the multi-path components from ray-tracing simulators. Based on this consent consideration, most of existing studies using stochastic geometry for $\mathrm{THz}$ networks concentrate on the mean interference power and mean SINR, rather than their distributions and coverage probability [9], [11], [17]. Specifically, an approach to the signal-to-interference ratio (SIR) distribution, proposed by [9], is to use a common log-logistic distribution to characterize the 


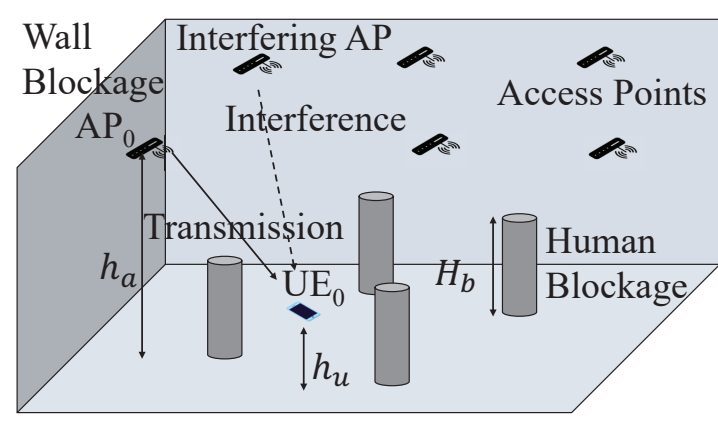

(a) The downlink of the indoor Tera-WLANs scenario in one room.

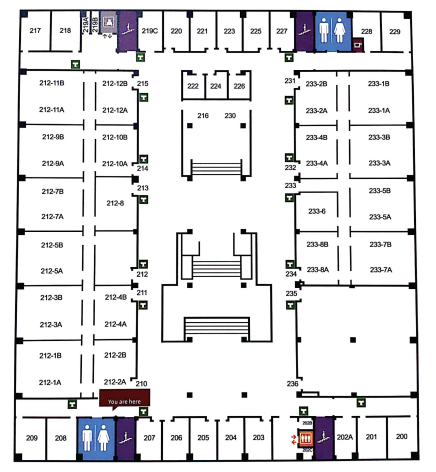

(b) The top vier
environment.

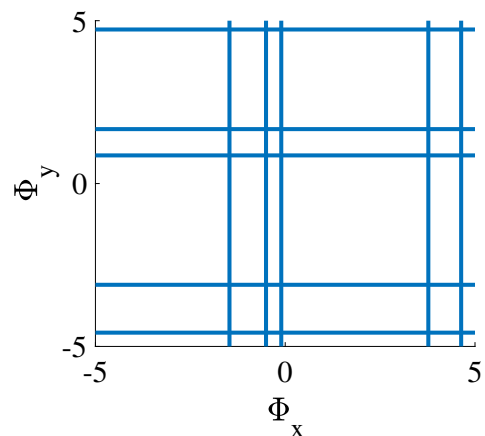

(c) An illustration of a MPLP wall blockage model.

Fig. 1. The system model of the Tera-WLANs.

interference distribution, which however requires parameter estimation with the simulation data to achieve the most accurate fitness. Petrov et al. [17] study the interference of $\mathrm{THz}$ networks with blocking and directional antennas, and derive the mean and variance of SINR by using a Taylor expansion approximation. In addition, the mean of the total interference is leveraged to define the SINR coverage probability in [11], which may not be reasonable, since the common definition is based on the instantaneous interference. Nonetheless, coverage analysis for $\mathrm{THz}$ networks is still an unsolved issue. Another open problem that attracts negligible attention in the literature when studying coverage is the impact of the $\mathrm{THz}$ user association scheme. A common assumption is that the user connects to the nearest $\mathrm{AP}$ as mostly inherited from microwave wireless systems. However, this consideration needs to be revisited in the THz networks, especially when the AP-UE link is possibly obstructed by the blockage [31].

In a nutshell, these aforementioned problems and challenges including 3D antenna patterns, indoor wall and human blockage, statistical channel modeling, and user association schemes, motive this study on indoor Tera-WLANs. In our preliminary and shorter version [1], we consider the human-body blockage and the nearest-AP user association, and derive the coverage probability of Terahertz wireless local area networks by proposing a modified log-normal interference model. In this paper, we further consider the impact of wall blockage on the interference region, which is another typical and important blocker in the indoor environment and has a significant impact on the radius of the interference region and the multi-path fading. In addition, we develop new approaches to compute coverage probability and average network throughput under the nearest-AP as well as LoS-AP user association schemes. Furthermore, much more extensive numerical results are provided to substantiate the interference, coverage and throughput analysis.

\section{INDOOR TERA-WLAN SYSTEM MODEL}

The downlink Tera-WLANs support wireless communications from APs to UEs in indoor scenarios, where multiple APs with transmitting power limited by $P_{t}$ are randomly mounted on the ceiling at the height of $h_{a}$ from the floor. Moreover, multiple UEs are randomly distributed on the user layer at the height of $h_{u}$, as shown in Fig. 1(a).

\section{A. Network Geometry}

We choose an arbitrary UE and assign it as the receiver (Rx) of interest, $\mathrm{UE}_{0}$. As is shown in Fig. 1(a), we consider a random deployment of APs modeled by a two-dimensional homogeneous Poisson point process (PPP) $\Phi_{a}$ with the density of $\lambda_{a}$. As the tagged UE, $\mathrm{UE}_{0}$ is associated with the tagged $\mathrm{AP}, \mathrm{AP}_{0}$. In the indoor environment, due to the very high penetration loss in the $\mathrm{THz}$ band caused by the wall blockage, the signal transmission through the wall is neglected.

From the top view in Fig. 1(b), the indoor environment is usually divided into a number of subspaces by the walls with two orientation angles that are perpendicular to each other in most cases. In our work, we consider to employ Manhattan Poisson line processes (MPLPS) to describe the wall blockage model [32]. In particular, the walls are assumed to be oriented perpendicular to the coordinate axes and modeled as two independent MPLPs, whose centers are distributed according to two one-dimensional Poisson point processes $\Phi_{x}$ and $\Phi_{y}$ with the same density $\lambda_{W}$ along $\mathrm{x}$-axis and $\mathrm{y}$-axis, as shown in Fig. 1(c). The tagged UE, $\mathrm{UE}_{0}$, and its associated AP and interfering APs are all in the same room. However, since the position of the tagged UE in the room is randomly distributed and the distribution between it and the associated AP is hard to obtain, the coverage probability at the tagged UE is intractable to analyze. Next, we propose to approximate the rectangleshaped interference region as a circle area, by treating the wall-blocking probability as a step function.

\section{B. Interference Region Modeling}

The direct link between the AP and the UE is blocked if at least one wall obstructs between them, i.e., there is at least one wall blocker that appears between the AP and the UE. We derive the wall-blocking probability for an arbitrary APUE link as

$$
\begin{aligned}
p_{W}(u, \theta) & =1-\mathbb{P}[\text { No wall blocker between } \mathrm{AP} \text { and } \mathrm{UE}] \\
& \stackrel{(a)}{=} 1-e^{-\lambda_{W} u(|\cos \theta|+|\sin \theta|)},
\end{aligned}
$$




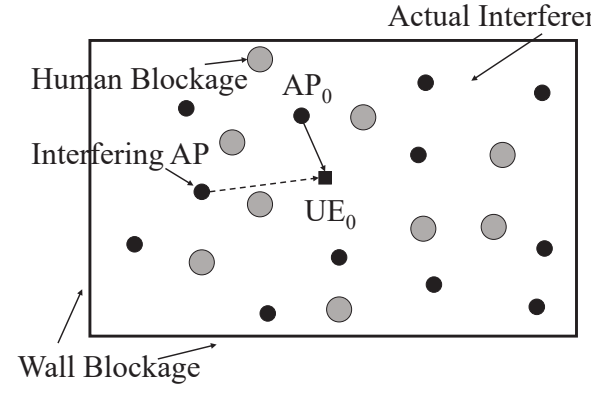

(a) The shape of an actual interference region.

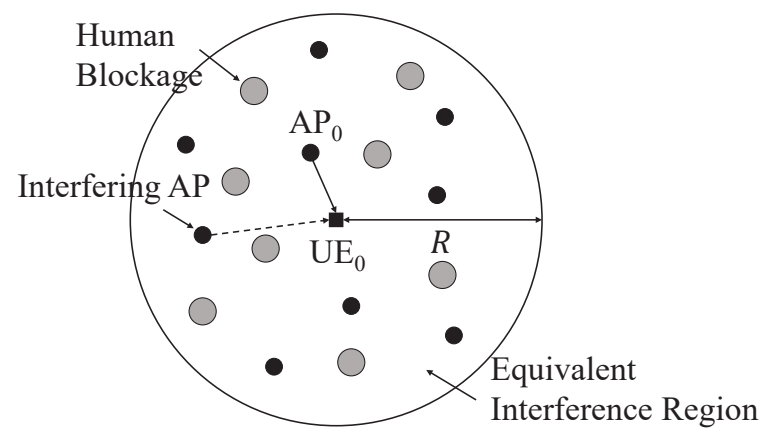

(b) Approximate interference region: an equivalent circle.

Fig. 2. Simplification of the rectangular interference region as an equivalent fixed circle. In (a), we show one realization of randomly located APs and wall blockers around the tagged UE. In (b), we model the interference region as a fixed circle. Only the signals from the APs inside the circle at the tagged $\mathrm{UE}$ are considered.

where $u$ denotes the 2D distance between the $\mathrm{AP}$ and the $\mathrm{UE}$, and $\theta$ refers to the azimuth angle, and 1(a) follows from the void probability of PPPs. While the wall-blocking probability for an AP-UE link is tractable to obtain, the wall blockage does not affect each link independently, i.e., the numbers of wall blockers on different links are not independent. If one wall blocker happens to locate among the two APs and the user, both of these two links are blocked at the same time. Therefore, the tractability of analysis is not maintained by directly using (1).

Instead, in order to maintain the tractability of coverage analysis, we propose to approximate the wall-blocking probability $p_{W}(u, \theta)$ by a step function [33], which is denoted as $S_{R}(u)$ that is equal to 0 when $0<u<R$ and 1 otherwise. Essentially, the wall-blocking probability of the link is considered as one outside a certain fixed radius $R$ and zero within the radius. This approximation is interpreted in Fig. 2, where the actual interference region is the rectangle geometry in Fig. 2(a) and replaced with the equivalent circle-shaped area in Fig. 2(b). The usage of the constant-size circle is actually a popular method to model the $\mathrm{THz}$ network as a finite zone with a fixed radius [11], [17]. The radius of the interference region is determined according to various criterion, e.g., the nodes outside the interference region provide negligible interference (much less than the noise power) and $R$ can be computed using the propagation model [17]. We propose to determine $R$ according to the criterion that the average number of the APs in the equivalent circle is equal to that in the actual rectangular interference region. According to the mean of a

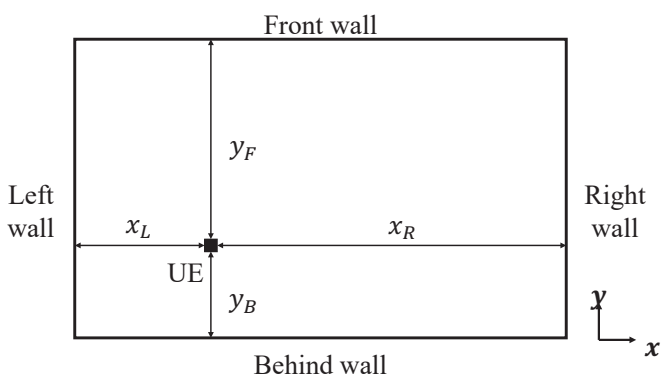

Fig. 3. An arbitrary UE is selected and located at a room that is composed of the right, left, front and behind walls around it.

Poisson distribution, when the wall-blocking probability is $S_{R}(u)$, the average number of APs around the tagged UE that are not blocked by walls is $\rho_{1}=\pi \lambda_{a} R^{2}$.

Lemma 1: In a rectangular region of Fig. 3 , the surrounding wall blockers follow the MPLPs. The 2D distances between an arbitrary user and the right, left, front and behind walls are denoted by $x_{R}, x_{L}, y_{F}$ and $y_{B}$, respectively, and all follow the exponential distribution, i.e., $x_{R}, x_{L}, y_{F}, y_{B} \sim \operatorname{Exp}\left(\lambda_{W}\right)$, according to the definition of the Poisson process.

We denote the number of the APs in the rectangular region as $S_{N}$, then the average number of the APs in the rectangular region is derived as

$$
\begin{aligned}
\rho_{2} & =\mathbb{E}\left[S_{N}\right] \\
& \stackrel{(a)}{=} \mathbb{E}_{x_{R}, x_{L}, y_{F}, y_{B}}\left[\lambda_{a}\left(x_{R}+x_{L}\right)\left(y_{F}+y_{B}\right)\right] \\
& \stackrel{(b)}{=} \frac{4 \lambda_{a}}{\lambda_{W}^{2}},
\end{aligned}
$$

where 2(a) follows from the mean of Poisson distribution, and 2(b) is obtained due to the fact that the sum of two exponential random variables with the rate parameter $\lambda_{W}$ is a Gamma random variable with the shape parameter 2 and the rate parameter $\lambda_{W}$. With the criterion of mean AP number, we have $\rho_{1}=\rho_{2}$, and obtain the radius of the circle-shaped region, $R=\frac{2}{\sqrt{\pi} \lambda_{W}}$.

Last, we describe the physical meaning of the simplification of the interference region. As shown in Fig. 2(a), the wall blockers are randomly located and the actual interference region for a UE has a rectangular shape with the random length and width. While we can obtain the wall-blocking probability for one AP-UE link (1), different links between different APs and the user are correlated, since they may share common wall blockages, which makes the network performance analysis hard to analyze. In Fig. 2(b), by introducing the step function, the interference region is equivalently approximated by a fixed ball $\mathcal{B}(0, R)$ with the radius of $R$. In this case, $\mathrm{UE}_{0}$ is located at the origin without any loss of generality, which is also a standard approach using the stochastic geometry method [15]. Meanwhile, the criterion determining the radius $R$ ensures the invariant of the average AP number. Essentially, we consider only one room or hall as the interference region, which may contain a number of THz APs, i.e., multiple cells. This is motivated by the fact that the Tera-WLANs are denser than current wireless networks. 


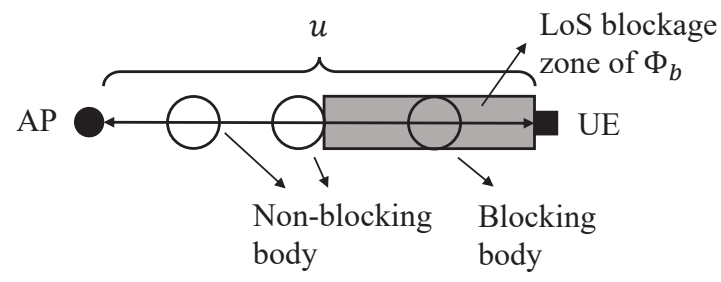

(a) Top view.

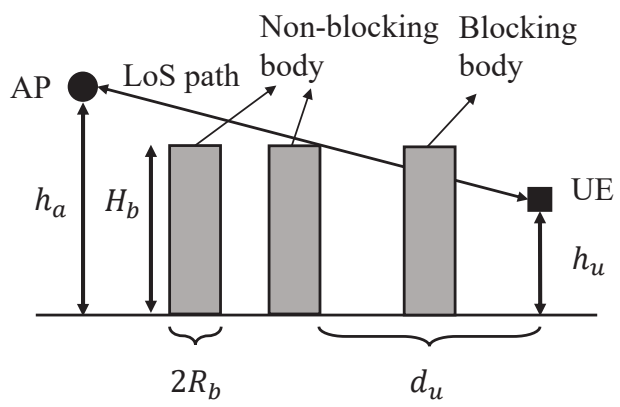

(b) Vertical view.

Fig. 4. Top and vertical views of the human-blocking scenario for an APUE link. The human body inside the LoS blockage zone in the top view corresponds to the rightmost body in the vertical view, and blocks the LoS path from the to and the UE.

\section{Human Blockage Model}

Another common blockage is a human body, which can be modeled as a cylinder with a radius of $R_{b}$ and a height of $H_{b}$. Since people stand randomly on the floor, we model the human blockage as a random circle process [24], [25], [34], essentially the bottom center of the cylinder as a two-dimensional homogeneous PPP with intensity of $\lambda_{b}$. As illustrated in Fig. 4, when the human body is inside the LoS blockage zone of $\Phi_{b}$, the LoS path of the AP-UE link is blocked. By contrast, human bodies that lie outside the LoS blockage zone belong cause no obstruction.

Lemma 2: The probability that an AP-UE link is blocked by a human body is $p_{B}(u)=1-e^{-\beta u}$, where $u$ is the $2 \mathrm{D}$ distance and $\beta=2 \lambda_{b} R_{b}\left(H_{b}-h_{u}\right) /\left(h_{a}-h_{u}\right), h_{a}$ and $h_{u}$ represent the heights of the AP and the UE from the floor [1].

\section{Blockage Effects on THz Link Types: LoS or NLoS}

Due to the very high penetration loss at the $\mathrm{THz}$ band, when the LoS path is blocked, the UE can only communicate with the AP via NLoS paths. On one hand, when the blockage is a human body, the signal transmission can be realized by the NLoS rays reflected on the walls of the room where the AP and the UE are located. On the other hand, if the blockage is a wall, i.e., the AP and the UE are located in different rooms, the signal transmission is neglected, since there is no NLoS path and severe loss in the channel through walls. Therefore, for the tagged UE, we divide the APs inside the circle-shape interference region into the LoS AP and the NLoS AP. Correspondingly, the point process $\Phi_{a}$ inside the interference region $\mathcal{B}(0, R)$ can be split into two thinning point processes, $\Phi_{L}$ and $\Phi_{N}$. The LoS probability is given by $p_{L}(u)=\left(1-S_{R}(u)\right)\left(1-p_{B}(u)\right)=e^{-\beta u}$ and the NLoS

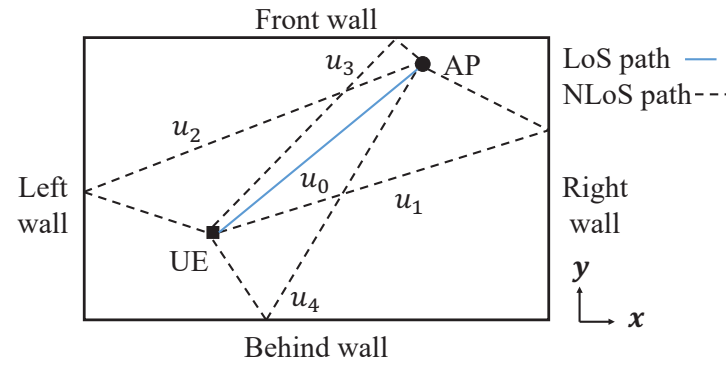

Fig. 5. Multi-ray THz channel model for indoor scenario: the NLoS rays are reflected by the walls.

probability is $p_{N}(u)=\left(1-S_{R}(u)\right) p_{B}(u)=1-e^{-\beta u}$ for $u<R$, where $u$ is the $2 \mathrm{D}$ distance between the AP and $\mathrm{UE}_{0}$.

The rate parameter of the exponential blockage model is determined by the density and the average size of the considered blockages [16]. At low frequencies, the signal is blocked by the randomly located buildings with a large size. The $\mathrm{THz}$ signal is easily blocked by the blockage of small size and the non-blocking probability decreases faster as the transmission distance, which results in a larger value of the rate parameter.

\section{E. User Association Schemes}

Two user association schemes are taken into account and comparison for the Tera-WLANs, which are listed as follows.

- Nearest-AP User Association: the user selects the nearest AP to associate with and the interfering AP are all further than the associated AP.

- LoS-AP User Association: the user selects the nearest LoS AP to associate with. If the LoS link is blocked by the moving blockages, the user switches to another LoS AP.

The nearest-AP association is based on the fact that the LoS probability is maximized as the dynamic human blockages move, since the LoS probability is a decreasing function with the 2D distance. Due to high reflection loss in the $\mathrm{THz}$ band, the nearest LoS AP is more likely to provide better SINR than the nearest AP that is NLoS. Motivated by this, we propose the LoS-AP association scheme for $\mathrm{THz}$ networks, and compare it with the classic nearest-AP scheme.

\section{Statistical THz Channel And Antenna Pattern MODELING}

\section{A. Multi-ray $\mathrm{THz}$ Channel Model}

The total attenuation of EM wave propagation in the $\mathrm{THz}$ band is composed of the molecular absorption and the free space path loss. The channel transfer function of the free space path loss is $H_{\text {spr }}(r)=\frac{c}{4 \pi f r}$, and the transfer function due to the molecular absorption is given by $H_{\mathrm{abs}}(r)=e^{-\frac{1}{2} \kappa(f) r}$, where $c$ refers to the speed of light, $f$ denotes the carrier frequency, $r$ is the propagation distance and $\kappa(f)$ stands for the frequency-dependent absorption coefficient [7].

As demonstrated in Fig. 5, we propose a multi-ray $\mathrm{THz}$ channel model for indoor environments by using deterministic ray-tracing techniques [8]. For an AP-UE link in a room, the 
multi-ray propagation model is expressed as a superposition of one LoS ray and $N_{R}$ NLoS rays reflected or scattered by the surrounding walls in the room [35]. We neglect the paths reflected by the ceiling and the floor due to the following reasons. First, unlike the flat walls, the ceiling is likely to be irregular and may not provide stable and reliable transmission. Second, from the perspective of antenna pattern, upward radiation is required if the NLoS ray reflected by the ceiling is considered. Third, since the APs in the considered scenario are mounted on the ceiling and very close to it, the NLoS ray will be also blocked if the LoS ray is blocked. As for the floor reflection, the reflection point on the floor is lower than all objects in the indoor environment and the UEs are usually on the table or in human's hand. In this case, the NLoS ray reflected by the floor can be easily obstructed. The 2D distances for the LoS path and the NLoS paths are denoted as $u_{0}$ and $u_{i}$, respectively. The azimuth between the UE and the AP is $\theta$. Hence, the channel response of the multi-ray model for the AP-UE link is

$H\left(u_{0}\right)=H_{\mathrm{LoS}}\left(u_{0}\right) e^{-j 2 \pi f \tau_{\mathrm{LoS}}} \mathbb{I}_{\mathrm{LoS}}+\sum_{i=1}^{N_{R}} H_{\mathrm{NLoS}}\left(u_{i}\right) e^{-j 2 \pi f \tau_{i}}$,

where $H_{\mathrm{LoS}}=H_{\mathrm{spr}}\left(v_{0}\right) H_{\mathrm{abs}}\left(v_{0}\right)$ and $H_{\mathrm{NLoS}}=$ $H_{\text {spr }}\left(v_{i}\right) H_{\text {abs }}\left(v_{i}\right) R_{c}(f)$ are the attenuation functions for the LoS ray and the NLoS rays, and $v_{i}=\sqrt{u_{i}^{2}+\left(h_{a}-h_{u}\right)^{2}}$ gives the $3 \mathrm{D}$ distance, $R_{c}(f)$ stands for the reflection coefficient that depends on the frequency and the material of the wall as well as the angle of incidence to the wall, $\tau_{\text {LoS }}=\frac{v_{0}}{c}$ and $\tau_{i}=\frac{v_{i}}{c}$ denote the LoS and NLoS path delays, respectively. Moreover, $\mathbb{I}_{\mathrm{LOS}}$ is an indicator function that is equal to 1 when the LoS path is blocked or 0 otherwise. Inspired by [8], we model the approximate reflection coefficient in $\mathrm{dB}$ unit as an independent normal random variable, $R_{c}[\mathrm{~dB}] \sim \mathcal{N}\left(\mu_{R}, \sigma_{R}^{2}\right)$. As a result, the reflection coefficient essentially follows a log-normal distribution. Due to the randomness of distance between the UE and the walls as discussed in Lemma 1, the multi-ray channel response is a random variable. Thus, the path loss that describes the attenuation of signal power, $L\left(u_{0}\right)=\left|H\left(u_{0}\right)\right|^{2}$, follows a random distribution.

Lemma 3: In the absence of the LoS path, the mean of $L\left(u_{0}\right)$ is given by

$$
\begin{aligned}
\mathbb{E}\left[L\left(u_{0}\right)\right] & =\frac{c^{2} \mathbb{E}\left[R_{c}^{2}\right]}{4 \pi^{2} f^{2}\left(u_{0}^{2}+\left(h_{a}-h_{u}\right)^{2}\right)} \\
& \cdot \frac{1}{\left(1+\ln \left(1+\frac{4}{\left(u_{0}^{2}+\left(h_{a}-h_{u}\right)^{2}\right) \lambda_{W}^{2}}\right)\right)},
\end{aligned}
$$

where $\mathbb{E}\left[R_{c}^{2}\right]=\exp \left(2 \frac{\ln 10}{10} \mu_{R}+2\left(\frac{\ln 10}{10} \sigma_{R}\right)^{2}\right)$ is the second moment of the reflection coefficient.

Proof: The proof is provided in Appendix A.

Lemma 4: In the presence of the LoS path, the mean of
$L\left(u_{0}\right)$ is derived as

$$
\begin{aligned}
\mathbb{E}\left[L\left(u_{0}\right)\right] & =\frac{c^{2}}{16 \pi^{2} f^{2}\left(u_{0}^{2}+\left(h_{a}-h_{u}\right)^{2}\right)} \\
& \cdot\left(1+\frac{4 \mathbb{E}\left[R_{c}^{2}\right]}{1+\ln \left(1+\frac{4}{\left(u_{0}^{2}+\left(h_{a}-h_{u}\right)^{2}\right) \lambda_{W}^{2}}\right)}\right),
\end{aligned}
$$

and the variance is given by

$$
\begin{aligned}
\operatorname{var}\left[L\left(u_{0}\right)\right] & =\frac{c^{4}}{256 \pi^{4} f^{4}\left(u_{0}^{2}+\left(h_{a}-h_{u}\right)^{2}\right)^{2}} \\
& \cdot\left(2+\frac{4 \mathbb{E}\left[R_{c}^{2}\right]}{1+\ln \left(1+\frac{4}{\left(u_{0}^{2}+\left(h_{a}-h_{u}\right)^{2}\right) \lambda_{W}^{2}}\right)}\right) \\
& \cdot \frac{4 \mathbb{E}\left[R_{c}^{2}\right]}{1+\ln \left(1+\frac{4}{\left(u_{0}^{2}+\left(h_{a}-h_{u}\right)^{2}\right) \lambda_{W}^{2}}\right)} .
\end{aligned}
$$

Proof: The proof is provided in Appendix A.

\section{B. Statistical Indoor THz Channel Model}

The multi-ray channel model accurately models the EM wave propagation, which however induces very high computational complexity and requires geometric information, making it difficult to be used in the network analysis. Instead, we develop an equivalent statistical $\mathrm{THz}$ channel model for the indoor $\mathrm{THz}$ networks. In this model, the power loss for the EM wave propagation in the $\mathrm{THz}$ band is described by the large-scale fading and the small-scale fading

$$
L\left(u_{0}\right)=K\left(u_{0}\right) g\left(u_{0}\right),
$$

where the large-scale fading $K\left(u_{0}\right)$ equals to $K_{L}\left(u_{0}\right)=$ $\left|H_{\text {spr }}\left(v_{0}\right) H_{\text {abs }}\left(v_{0}\right)\right|^{2}$ for the case that the LoS path is present. On the contrary, the large-scale fading $K\left(u_{0}\right)$ boils down to $K_{N}\left(u_{0}\right)=\left|H_{\text {spr }}\left(v_{0}\right) H_{\text {abs }}\left(v_{0}\right)\right|^{2} \mathbb{E}\left[R_{c}^{2}\right]$ in the NLoS case. The path loss due to the small-scale fading caused by the multi-path effect, $g\left(u_{0}\right)$, follows a distribution with distancedependent parameters. In the LoS case, we assume that $g$ is a Gamma random variable with the shape parameter $a\left(u_{0}\right)$ and the rate parameter $b\left(u_{0}\right)$, which is expressed as $g_{L} \sim$ Gamma $(a, b)$, where $a=\frac{\mathbb{E}\left[L\left(u_{0}\right)\right]^{2}}{\operatorname{var}\left(L\left(u_{0}\right)\right)}, b=K_{L}\left(u_{0}\right) \frac{\mathbb{E}\left[L\left(u_{0}\right)\right]}{\operatorname{var}\left(L\left(u_{0}\right)\right)}$, and $\mathbb{E}\left[L\left(u_{0}\right)\right]$ and $\operatorname{var}\left(L\left(u_{0}\right)\right)$ follow from (5) and (6), respectively. In the NLoS case, $g$ is assumed to follow an exponential distribution with the rate parameter $\mu\left(u_{0}\right), g_{N} \sim$ $\operatorname{Exp}(\mu)$, where $\mu=\frac{K_{N}\left(u_{0}\right)}{\mathbb{E}\left[L\left(u_{0}\right)\right]}$ and $\mathbb{E}\left[L\left(u_{0}\right)\right]$ follows from (4).

The use of the Gamma distribution for the LoS case and the exponential distribution for the NLoS case is motivated by the following aspects. When there is no dominant propagation, the Rayleigh fading is a reasonable statistical channel model in the microwave band and the path loss is exponentially distributed. In the above multi-ray channel, the path loss of all the NLoS rays is considered to be identically and independently distributed. Naturally, we propose an exponential distribution statistical model with a distance-dependent parameter for the NLoS transmission. When the LoS ray is not blocked, we 


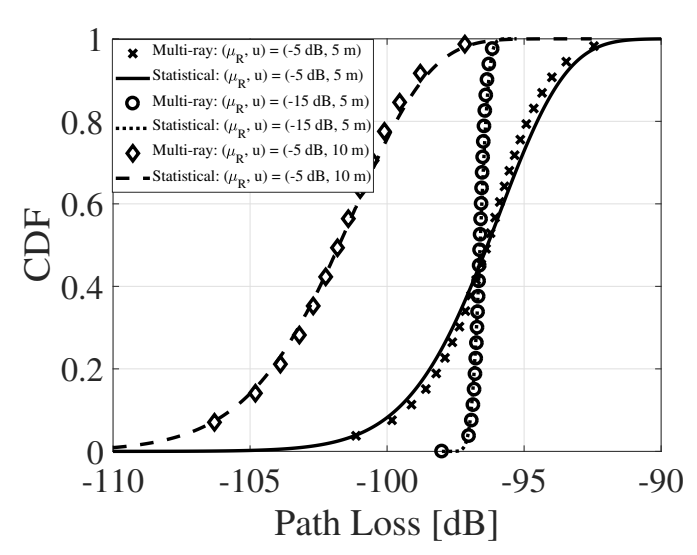

Fig. 6. Comparison between the multi-ray model and the equivalent statistical model: LoS case.

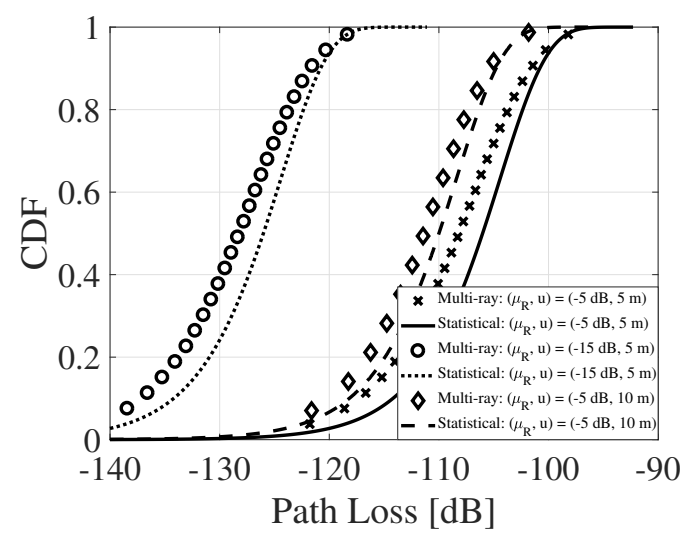

Fig. 7. Comparison between the multi-ray model and the equivalent statistical model: NLoS case.

use a Gamma distribution model to characterize the smallscale fading of the LoS transmission, which can be seen as a modified channel model of Nakagami fading that is also commonly used in the microwave band.

\section{Accuracy of the Proposed Statistical Model}

We give some numerical results on the cumulative probability function (CDF) of the path loss to validate the accuracy of the statistical channel model, as a comparison of the multiray model. The parameters of the reflection coefficient are $\mu_{R}=-5,-15 \mathrm{~dB}$ and $\sigma_{R}=2 \mathrm{~dB}$. We can observe that the CDFs of these two models match well for both LoS case in Fig. 6 and NLoS case in Fig. 7. The statistical model accurately characterizes the small-scale fading caused by the multi-path propagation and also maintains the tractability of stochastic analysis, which the multi-ray model lacks. From Fig. 6 and Fig. 7, we can see that the path loss becomes stronger as the $2 \mathrm{D} \mathrm{LoS}$ propagation distance varies from $5 \mathrm{~m}$ to $10 \mathrm{~m}$, which determines the variation of the large-scale fading. For the LoS case, when the reflection loss is very strong, e.g., $\mu_{R}$ is $-15 \mathrm{~dB}$, the small-scale fading can be ignored, as the power of the LoS ray is much higher than the NLoS rays. But for the NLoS case, small-scale fading is supposed to be considered no matter how much the reflection loss is.

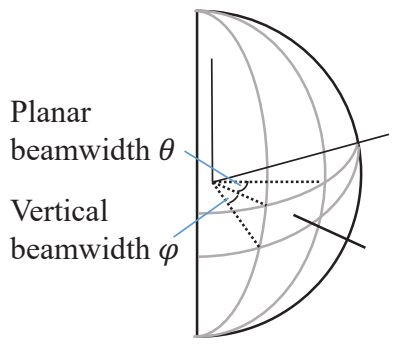

(a)

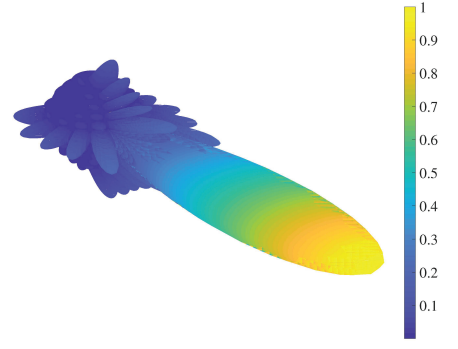

(b)
Fig. 8. Illustration of the antenna beam model: (a) A pyramidal shape model of the antenna beam. (b) The simulated antenna beam.

By comparing Fig. 6 and Fig. 7, we show that closer NLoS AP with the $2 \mathrm{D}$ distance of $5 \mathrm{~m}$ to the user can have stronger path loss than the LoS AP with the longer distance of $10 \mathrm{~m}$, indicating that in the Tera-WLANs the AP-UE distance is not the top priority when the user selects an AP to connect. Hence, the LoS-AP user association is expected to provide better coverage probability than the nearest-AP user association in the THz networks.

\section{3D Antenna Radiation Model}

In our work, directional 3D beams are used at both APs and UEs to compensate the severe path loss in the THz band. We consider the LoS ray and each of the reflected rays is radiated through one antenna beam with the same antenna gain, i.e., the number of rays is equal to the number of beams. The radiation of one antenna beam is modeled as a pyramidal shape with the planar beamwidth $\theta$ and the vertical beamwidth $\varphi$ [19], [36], as plotted in Fig. 8. The pyramidal model has a similar shape with the simulated antenna radiation beam using multiple antennas. By using the pyramidal model for the antenna pattern, we are able to obtain an approximated relationship between the beamwidth and the antenna gain, and analyze if the UE is within the planar beam and the vertical beam of the AP individually. The antenna gain as a function of the angles $\theta$ and $\varphi$ is given by [19]

$$
G_{\mathrm{MB}}(\theta, \varphi)=\frac{\pi}{\arcsin \left(\tan \frac{\theta}{2} \tan \frac{\varphi}{2}\right)},
$$

In particular, the APs and the UEs have different antenna pattern parameters due to the different hardware requirements. The planar beamwidth and the vertical beamwidth at the APs and the UEs are denoted as $\theta_{a}, \varphi_{a}$ and $\theta_{u}, \varphi_{u}$, respectively. The APs usually are equipped with more antennas that result in a narrower beamwidth and higher antenna gains. For the directions outside the pyramidal, the side lobe gains are over $10 \mathrm{~dB}$ less than that of the main beam. Therefore, the radiation from the side lobe is neglected in the following analysis.

\section{INTERFERENCE ANALYSIS}

The SINR at the tagged UE is expressed as

$$
\mathrm{SINR}=\frac{S}{I+P_{N}}=\frac{P_{t} G_{0} K(u) g_{0}(u)}{\sum_{i \in \Phi_{a} / \mathrm{AP}_{0}} P_{t} G_{i} K\left(x_{i}\right) g_{i}\left(x_{i}\right)+P_{N}},
$$




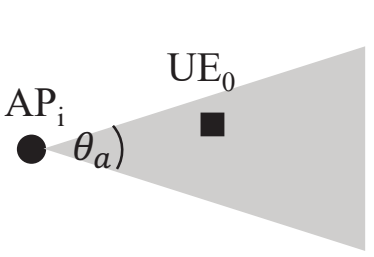

(a) Top view

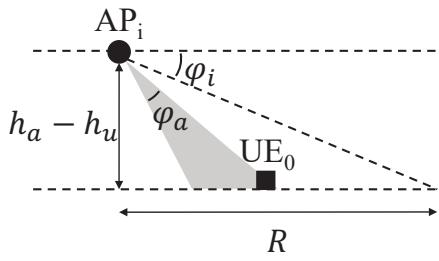

(b) Vertical view.
Fig. 9. Antenna gain for interfering APs.

where $P_{t}$ refers to the transmit power, $G_{0}$ represents the antenna gain of the received signal power, $u$ is the $2 \mathrm{D}$ distance between the tagged UE and its associated AP, and $x_{i}, G_{i}$ are the horizontal distances between the $i^{\text {th }}$ AP and the tagged UE and the corresponding antenna gain. Additionally, $P_{N}=N_{0} B$ computes the Johnson-Nyquist noise power, where $N_{0}$ describes the noise power spectral density (PSD), and $B$ is the bandwidth.

We can see that a key step to obtain the SINR distribution is to analyze the aggregated interferences. Therefore, we conduct interference analysis under the two user association schemes in our work. Before that, we need to study the horizontal distance between the tagged UE and its associated AP, which has correlation with the location distribution of interferers.

\section{A. Distribution of Horizontal Distance Between the UE and the Associated AP}

1) Nearest-AP User Association: The probability density function of $u$ is given by [15],

$$
f_{1}(u)=2 \pi \lambda_{a} u e^{-\lambda_{a} \pi u^{2}}, 0 \leq u \leq R .
$$

2) LoS-AP User Association: The probability that the user has at least one LoS AP is

$$
\begin{aligned}
A_{L} & =1-e^{-2 \pi \lambda_{a} \int_{0}^{R} p_{L}(u) u d u} \\
& =1-e^{-\frac{2 \pi \lambda_{a}}{\beta^{2}}(1-\exp (-\beta R)-\beta R \exp (-\beta R))},
\end{aligned}
$$

where $\beta=2 \lambda_{b} R_{b}\left(H_{b}-h_{u}\right) /\left(h_{a}-h_{u}\right)$ refers to the rate parameter of the human-blocking probability. With the LoSAP user association scheme, the probability that the user is associated with a LoS AP is equal to $A_{L}$. Given that the user is associated with a $\operatorname{LoS} \mathrm{AP}$, the PDF of the $2 \mathrm{D}$ distance to its associated AP is

$$
\begin{aligned}
f_{2}(u) & =2 \pi \lambda_{a} p_{L}(u) u e^{-2 \pi \lambda_{a} \int_{0}^{u} p_{L}(x) x d x} / A_{L} \\
& =2 \pi \lambda_{a} u e^{-\beta u-\frac{2 \pi \lambda_{a}}{\beta^{2}}(1-\exp (-\beta u)-\beta u \exp (-\beta u))} / A_{L} .
\end{aligned}
$$

\section{B. Analysis for Antenna Gains of Interfering Signals}

The antenna gain of the transmission signal is the product of the transmitting and receiving antenna gains, denoted by $G_{\mathrm{Tx}} G_{\mathrm{Rx}}$. Since the antenna beams of the tagged UE and its associated AP point towards each other, the transmitting and receiving antenna gains of the received signal at the tagged UE should both equal to their main lobe gains, given by $G_{\mathrm{Tx}}=$ $G_{a}=G_{\mathrm{MB}}\left(\theta_{a}, \varphi_{a}\right)$, and $G_{\mathrm{Rx}}=G_{u}=G_{\mathrm{MB}}\left(\theta_{u}, \varphi_{u}\right)$.
By contrast, for the interfering signal, the antenna gain is approximately equal to $G_{\mathrm{MB}}$ with some probabilities. $G_{\mathrm{Tx}}$ depends on whether the tagged UE is within the transmitting antenna beam from the interfering AP, while $G_{\mathrm{Rx}}$ depends on whether the interfering AP is within the receiving antenna beam of the tagged UE.

We take the transmitting antenna gain between $\mathrm{AP}_{\mathrm{i}}$ and the tagged UE as an example first, as demonstrated in Fig. 9. We consider that the depression angle from $\mathrm{AP}_{0}$ to its associated user is uniformly distributed between $\varphi_{i}$ and $\frac{\pi}{2}$, where $\varphi_{i}=\arctan \left(\frac{h_{a}-h_{u}}{R}\right)$ is the depression angle from $\mathrm{AP}_{0}$ to the average cell bound with the radius of $R$. The transmitting antenna gain is equal to the main beam gain with the probability $p_{A}=p_{H} p_{V}$, where $p_{H}=\frac{\theta_{a}}{2 \pi}$ is the probability when $\mathrm{UE}_{0}$ is within the planar beam of $\mathrm{AP}_{\mathrm{i}}$ and $p_{V}=\min \left\{\frac{\varphi_{a}}{\frac{\pi}{2}-\varphi_{i}}, 1\right\}$ is the probability that $\mathrm{UE}_{0}$ is within the vertical beam of $\mathrm{AP}_{\mathrm{i}}$. The antenna gain of the interfering signal at the tagged UE is equal to the gain of the main beam with the approximate probability of the beamwidth over all possible angles $\left(2 \pi\right.$ from the top view and $\frac{\pi}{2}-\varphi_{i}$ from the vertical view) [16], [17].

For the receiving antenna gain, we assume that the vertical beamwidth of the UE antenna $\varphi_{u}$ is relatively wide so that $\mathrm{AP}_{\mathrm{i}}$ is within the vertical beam of $\mathrm{UE}_{0}$ only if $\mathrm{AP}_{\mathrm{i}}$ is within the planar beam. Thus, the receiving antenna gain is equal to the main beam gain with the probability $p_{U}=\frac{\theta_{u}}{2 \pi}$. Therefore, the distribution of the antenna gains $G$ for the signal between the interfering APs and the tagged UE is $G_{a} G_{u}$ with the probability $p_{A} p_{U}$ and zero with the probability $1-p_{A} p_{U}$. To be precise, the above analysis is aimed at the LoS transmission. For the tractability of analysis, we assume that the antenna gains for NLoS links have the identical distribution as the LoS link.

\section{Interference Analysis}

1) Nearest-AP User Association: In this user association scheme, all interferers are farther than the associated AP, i.e., their horizontal distances are larger than $u$. Thus, the aggregated interference at the tagged UE in the network is expressed as $I_{\text {aggr }}=\sum_{i \in \Phi_{a} / \mathrm{AP}_{0}} P_{t} G_{i} K\left(x_{i}\right) g_{i}\left(x_{i}\right), u \leq x_{i} \leq R$ where $x_{i}$ is the 2D distance of the $i^{\text {th }}$ interfering AP (except the associated $\mathrm{AP}$ for the tagged UE denoted by $\mathrm{AP}_{0}$ ) from the tagged UE, and $g_{i}, G_{i}$ are defined as the channel fading coefficient and the antenna gain and have independent identical distributions for all $i$.

Since the literature [15], [16] has suggested that closed-form expression for the interference distribution is intractable, we pay our attention to the moments of the interference power, especially the mean interference power. In order to evaluate the moments of the aggregated interference, we first derive the moment generating function of the interference, which depends on the $2 \mathrm{D}$ distance between $\mathrm{AP}_{0}$ and $\mathrm{UE}_{0}$, and yield (13) by using the definition of MGF. While the solution of this equation contains the integral over the distances, it is still faster and more convenient to conduct numerical computation than the complete Monte-Carlo system simulation. Since the range of the integration in (13) starts from $u$, 


$$
\begin{aligned}
& \mathcal{M}_{I_{\text {aggr }}}(s)=\mathbb{E}_{\Phi_{a}}\left[\prod_{i \in \Phi_{a} / \mathrm{AP}_{0}} \mathbb{E}_{G, g}\left[\exp \left(s P_{t} G g\left(x_{i}\right) K\left(x_{i}\right)\right)\right]=\exp \left(-2 \pi \lambda_{a} \int_{u}^{R}\left(1-\mathbb{E}\left[e^{s P_{t} G K(x) g(x)}\right]\right) x d x\right)\right. \\
& =\exp \left(-2 \pi \lambda_{a} p_{A} p_{u} \int_{u}^{R}\left(\left(1-\left(1-\frac{s P_{t} G_{a} G_{u} K_{L}(x)}{b(x)}\right)^{-a(x)}\right) p_{L}(x)+\left(1-\frac{\mu(x)}{\mu(x)-s P_{t} G_{a} G_{u} K_{N}(x)}\right) p_{N}(x)\right) x d x\right) .
\end{aligned}
$$

the MGF of the aggregated interference depends on the 2D distance between $\mathrm{UE}_{0}$ and $\mathrm{AP}_{0}$. Therefore, when deriving the moments of the interference power, we need to obtain the conditional moments and then take the expectation on $u$. The $n^{\text {th }}$ moment of the aggregated interference can be calculated as $\mathbb{E}\left[I^{n} \mid u\right]=\left.\frac{d^{n}}{d s^{n}} \mathcal{M}_{I}(s)\right|_{s=0}$. Therefore, the conditional mean interference power becomes

$$
\begin{aligned}
\mathbb{E}\left[I_{\mathrm{aggr}} \mid u\right] & =2 \pi \lambda_{a} p_{A} p_{U} G_{a} G_{u} P_{t} \int_{u}^{R}\left(\frac{a(x)}{b(x)} K_{L}(x) p_{L}(x)\right. \\
& \left.+\frac{K_{L}(x)}{\mu(x)} p_{N}(x)\right) x d x .
\end{aligned}
$$

Then the mean interference power is given by

$$
\begin{aligned}
\mathbb{E}\left[I_{\mathrm{aggr}}\right] & =4 \pi^{2} \lambda_{a}^{2} p_{A} p_{U} G_{a} G_{u} P_{t} \int_{0}^{R} \int_{u}^{R}\left(\frac{a(x)}{b(x)} K_{L}(x) p_{L}(x)\right. \\
& \left.+\frac{K_{L}(x)}{\mu(x)} p_{N}(x)\right) x d x u e^{-\lambda_{a} \pi u^{2}} d u .
\end{aligned}
$$

This analytical expression shows that the mean interference power is proportional to $p_{A} p_{U} G_{a} G_{u}$, which is essentially the expected antenna gain, and the transmit power $P_{t}$. Approximately, it is also proportional to $\lambda_{a}^{2}$, which indicates that increasing the AP density leads to stronger mean interference power. In addition, the upper limit of (15) is determined by the radius $R$, which states that increasing the wall density $\lambda_{W}$ can reduce the upper limit and weaken the mean interference power. Moreover, since the integration function contains $p_{L}(x)$ and $p_{N}(x)$, the mean interference power is also affected by the human density $\lambda_{b}$. By increasing the human density, some LoS interfering APs might become NLoS due to the blockage and thus cause less interference power.

2) LoS-AP User Association: In this association scheme, the user is associated with a LoS AP and the horizontal AP-UE distance is $u$. In this case, the interferers can be categorized into two subsets, namely, the LoS APs $\Phi_{L}$ and the NLoS APs $\Phi_{N}$. On one hand, the interference power from the LoS APs is $I_{L}=\sum_{i \in \Phi_{L}} P_{t} G_{i} K_{L}\left(x_{i}\right) g_{L, i}\left(x_{i}\right), u \leq$ $x_{i} \leq R$, where $G_{i}=G_{\mathrm{Tx}, i} G_{\mathrm{Rx}, i}$ is the antenna gain of the interfering signal from the $i^{\text {th }} \operatorname{LoS}$ AP. On the other hand, the interference power from the NLoS APs is $I_{N}=$ $\sum_{i \in \Phi_{N}} P_{t} G_{i} K_{N}\left(x_{i}\right) g_{N, i}\left(x_{i}\right), 0 \leq x_{i} \leq R$. Similar to the derivation of (13), we can obtain the MGFs of the interference using the LoS-AP user association

$$
\mathcal{M}_{I_{L}}(s)=e^{-2 \pi \lambda_{a} \int_{u}^{R}\left(1-\left(1-\frac{s P_{t} G_{a} G_{u} K_{L}(x)}{b(x)}\right)^{-a(x)}\right) p_{L}(x) x d x},
$$

$$
\mathcal{M}_{I_{N}}(s)=e^{-2 \pi \lambda_{a} \int_{0}^{R}\left(1-\frac{\mu(x)}{\mu(x)-s P_{t} G_{a} G_{u} K_{N}(x)}\right) p_{N}(x) x d x} .
$$

Combining the LoS interference and the NLoS interference, we can obtain the total interference power is $I_{L}+I_{N}$, whose MGF is the product of $\mathcal{M}_{I_{L}}(s)$ and $\mathcal{M}_{I_{N}}(s)$. The main difference for the interference analysis between two association schemes lies in the integration range. For the nearest-AP user association, the integration range is from $u$ to $R$, which is the same as the LoS interference while using the LoS-AP association. However, in the latter scheme, the distribution of NLoS interfering APs is independent of the associated AP, which makes the integration range is from 0 to $R$. Following the same way as the nearest-AP user association scheme, the mean interference power or higher moments can be calculated when using the LoS-AP user association.

\section{Coverage And Network Throughrut}

The coverage probability $P_{c}(T)$ is defined as the probability that the received SINR at the tagged UE is larger than some SINR threshold $T>0$, i.e., $P_{c}(T)=\mathbb{P}(\operatorname{SINR}>T)$, which can be considered as the CCDF of SINR. According to the reflection loss, we propose two different approaches to coverage probability for two cases: low reflection loss $\left(\mu_{R}>-10 \mathrm{~dB}\right)$ and high reflection loss $\left(\mu_{R}<-10 \mathrm{~dB}\right)$.

\section{A. Coverage Probability: Case 1}

Based on the interference analysis in the last section, we first present the main results on the coverage probability for the first case as follows.

1) Nearest-AP User Association: With the nearest-AP user association scheme, the user is supposed to connect to the nearest AP in $\Phi_{a}$. The coverage probability $P_{c}(T)$ is

$$
P_{c}(T)=\int_{0}^{R}\left(p_{L}(u) P_{c, L}(T)+p_{N}(u) P_{c, N}(T)\right) f_{1}(u) d u
$$

where $P_{c, L}(T)$ is the conditional coverage probability given that the user connects to a LoS AP with the 2D distance $u$, and can be derived as

$$
\begin{aligned}
P_{c, L}(T) & =\mathbb{P}\left(g_{L}(u)>\frac{T\left(I_{\mathrm{aggr}}+P_{N}\right)}{P_{t} G_{a} G_{u} K_{L}(u)}\right) \\
& \stackrel{(a)}{\approx} 1-\left(1-e^{-\epsilon(u) b(u) \frac{T\left(I_{\mathrm{aggr}}+P_{N}\right)}{P_{t} G_{a} G_{u} K_{L}(u)}}\right)^{a(u)} \\
& \stackrel{(b)}{=} \sum_{n=1}^{\infty}(-1)^{n+1}\left(\begin{array}{c}
a(u) \\
n
\end{array}\right) e^{-\frac{n T P_{N} b(u) \epsilon(u)}{P_{t} G_{a} G_{u} K_{L}(u)}} \\
& \cdot \mathcal{M}_{I_{\text {aggr }}}\left(-\frac{n T b(u) \epsilon(u)}{P_{t} G_{a} G_{u} K_{L}(u)}\right),
\end{aligned}
$$




$$
\begin{aligned}
& P_{c}(T) \approx \int_{0}^{R_{1}}\left(P_{0}(0)+P_{1}(0) \int_{0}^{R} \frac{2 x p_{N}(x)}{R^{2}} d x\right) A_{L} f_{2}(u) d u+\int_{0}^{R_{2}} \int_{\max \left\{u, u_{x}\right\}}^{R} \frac{2 x p_{L}(x)}{R^{2}-u^{2}} d x P_{1}(u) A_{L} f_{2}(u) d u \\
& =\int_{0}^{R_{1}} 2 \pi \lambda_{a} u e^{-\beta u-\frac{2 \pi \lambda_{a}}{\beta^{2}}(1-\exp (-\beta u)-\beta u \exp (-\beta u))} e^{-\lambda_{a} p_{A} p_{U} \pi R^{2}}\left(1+\lambda_{a} p_{A} p_{U} \pi\left(R^{2}+2 R e^{-\beta R}+\frac{2 e^{-\beta R}}{\beta^{2}}-\frac{2}{\beta^{2}}\right)\right) d u \\
& +\int_{0}^{R_{2}} 2 \pi^{2} \lambda_{a} p_{A} p_{U} u e^{-\beta u-\frac{2 \pi \lambda_{a}}{\beta^{2}}(1-\exp (-\beta u)-\beta u \exp (-\beta u))} e^{-\lambda_{a} p_{A} p_{U} \pi R^{2}}\left(\frac{2 u_{m} e^{-\beta u_{m}}}{\beta}+\frac{2 e^{-\beta u_{m}}}{\beta^{2}}-\frac{2 R e^{-\beta R}}{\beta}-\frac{2 e^{-\beta R}}{\beta^{2}}\right) d u .
\end{aligned}
$$

where $\epsilon(u)=(\Gamma(1+a(u)))^{-\frac{1}{a(u)}}, 19$ (a) follows from the tight bound of the gamma distribution in [37], and 19(b) originates from the generalized Binomial theorem. The conditional coverage probability, given that the user connects to a NLoS AP with the $2 \mathrm{D}$ distance $u$, is given by

$$
\begin{aligned}
P_{c, N}(T) & =\mathbb{P}\left(g_{N}(u)>\frac{T\left(I_{\text {aggr }}+P_{N}\right)}{P_{t} G_{a} G_{u} K_{N}(u)}\right) \\
& =e^{-\frac{T P_{N}}{P_{t} G_{a} G_{u} K_{N}(u)}} \mathcal{M}_{I_{\text {aggr }}}\left(-\frac{T}{P_{t} G_{a} G_{u} K_{N}(u)}\right) .
\end{aligned}
$$

In the analytical expressions for the conditional coverage probabilities, (19) and (20), the MGF of the aggregated interference $\mathcal{M}_{I_{\text {aggr }}}(s)$ plays an important role, and the coverage probability is calculated by substituting $s=-\frac{n T b(u) \epsilon(u)}{P_{t} G_{a} G_{u} K_{L}(u)}$ in $P_{c, L}(T)$ and $s=-\frac{T}{P_{t} G_{a} G_{u} K_{N}(u)}$ in $P_{c, N}(T)$. Intuitively, increasing the AP density can not only improve the received signal power, but also enhance the interference power. Thus, it is unable to learn whether increasing the AP density can improve the coverage probability directly from the above analytical expressions. In addition, if we increase the antenna gain of the main beam by reducing the beam-width and keep the expected antenna gain unchanged, the coverage probability will be improved.

2) LoS-AP User Association: We propose a scheme, in which the user is associated with the nearest LoS AP in $\Phi_{L}$. The coverage probability $P_{c}(T)$ is given by $P_{c}(T)=$ $A_{L} P_{L}(T)$, where the coverage probability $P_{L}(T)$ given that the user is associated with a LoS AP is

$$
\begin{aligned}
& P_{L}(T)=\mathbb{P}(\operatorname{SINR}>T) \\
& =\int_{0}^{R} \mathbb{P}\left(g_{L}(u)>\frac{T\left(I_{L}+I_{N}+P_{N}\right)}{P_{t} G_{\mathrm{MB}}^{2} K_{L}(u)}\right) f_{2}(u) d u \\
& =\int_{0}^{R} f_{2}(u) \sum_{n=1}^{\infty}(-1)^{n+1}\left(\begin{array}{c}
a(u) \\
n
\end{array}\right) e^{-\frac{n T P_{N} b(u) \epsilon(u)}{P_{t} G_{\mathrm{MB}}^{2} K_{L}(u)}} \\
& \cdot \mathcal{M}_{I_{L}}\left(-\frac{n T b(u) \epsilon(u)}{P_{t} G_{\mathrm{MB}}^{2} K_{L}(u)}\right) \mathcal{M}_{I_{N}}\left(-\frac{n T b(u) \epsilon(u)}{P_{t} G_{\mathrm{MB}}^{2} K_{L}(u)}\right) d u .
\end{aligned}
$$

\section{B. Coverage Probability: Case 2}

The AP point process $\Phi_{a}$ in the interference region is divided into two independent non-homogeneous PPPs $\Phi_{L}$ and $\Phi_{N}$, which are considered as two independent tiers of the network. From Section IV-C, we learn that the path loss from the APs in the NLoS tier $\Phi_{N}$ to the user is almost always larger than the APs from the LoS tier regardless of the propagation distance, which indicates that the variation of the path loss in the THz networks is much more sensitive to the human blockage than the distance. Thus, we propose the LoS-AP user association scheme, which selects the associated AP in the LoS tier, different from the nearest-AP user association that does not consider the tier type of the associated AP.

In the case of strong reflection loss, since the power of NLoS ray becomes much lower than the LoS ray, the shape parameter of Gamma distribution in the statistical channel model becomes very large, which makes 19(a) not an accurate approximation. When the reflection loss is large, the signal power of the NLoS rays can be neglected, compared to that of the LoS ray. In this case, we propose a new tractable approach to coverage probability for the $\mathrm{THz}$ networks. First we define the dominant interferers as the APs with the antenna gain of the interfering signal equal to $G_{a} G_{u}$. We introduce the probability that the number of the dominant interfering APs in the region bounded by two concentric circles of radii $u(0<u<R)$ and $R$ is equal to $k$ as $P_{k}(u)=\frac{1}{k !} e^{-\lambda_{a} p_{A} p_{U} \pi\left(R^{2}-u^{2}\right)}\left(\lambda_{a} p_{A} p_{U} \pi\left(R^{2}-u^{2}\right)\right)^{k}$. Since $P_{0}(u)+P_{1}(u)$ is close to 1 when $\lambda_{a} p_{A} p_{U}$ is small, the number of the dominant interfering APs is not more than one with high probability close to one. Based on this fact, we derive the semi-closed-form expression of the coverage probability for high reflection loss with the LoS-AP user association as (22), where $R_{1}=\min \left\{R, R_{T}\right\}, R_{2}=\min \left\{R, R_{T}, R_{I}\right\}$, and $R_{T}=\sqrt{\max \left\{\left(\frac{c}{4 \pi f}\right)^{2} \frac{P_{t} G_{a} G_{u}}{T P_{N}}-\left(h_{a}-h_{u}\right)^{2}, 0\right\}}, R_{I}=$ $\sqrt{\max \left\{\frac{1}{T} \frac{1}{\frac{1}{R^{2}+\left(h_{a}-h_{u}\right)^{2}}+\left(\frac{4 \pi f}{c}\right)^{2} \frac{P_{N}}{P_{t} G_{a} G_{u}}}-\left(h_{a}-h_{u}\right)^{2}, 0\right\}}, \quad$ $u_{x}=\sqrt{\max \left\{\frac{1}{\frac{1}{T} \frac{1}{u^{2}+\left(h_{a}-h_{u}\right)^{2}}-\left(\frac{4 \pi f}{c}\right)^{2} \frac{P_{N}}{P_{t} G_{a} G_{u}}}-\left(h_{a}-h_{u}\right)^{2}, 0\right\}}$, $u_{m}=\max \left\{u, u_{x}\right\}$. The derivation of the coverage probability with the nearest-AP user association follows the similar way and is omitted here due to the limited space. When $\beta=0$, the two user association schemes become the same and we obtain the following closed-form expression (23), which can be viewed as a function the AP density, $g\left(\lambda_{a}\right)$. From (23) we learn that the coverage probability is increased at first and then reduced, as the AP density $\lambda_{a}$ becomes larger.

Furthermore, we formulate a optimization problem of calculating the optimal AP density as

$$
\max _{\lambda_{a}} g\left(\lambda_{a}\right) \quad \text { s.t. } \quad \lambda_{a}>0
$$

which is a convex optimization problem and can be solved very efficiently. 


$$
\begin{aligned}
P_{c}(T) & =e^{-\lambda_{a} p_{A} p_{U} \pi R^{2}} \frac{1-e^{-\left(1-p_{A} p_{U}\right) \pi \lambda_{a} R_{1}^{2}}}{1-p_{A} p_{U}}+\lambda_{a} \pi p_{A} p_{U} e^{-\lambda_{a} p_{A} p_{U} \pi R^{2}} \frac{1-e^{-\left(1-p_{A} p_{U}\right) \pi \lambda_{a} R_{2}^{2}}}{1-p_{A} p_{U}}\left(R^{2}-(T-1)\left(h_{a}-h_{u}\right)^{2}\right) \\
& -T p_{A} p_{U} e^{-\lambda_{a} p_{A} p_{U} \pi R^{2}}\left(\frac{1-e^{-\left(1-p_{A} p_{U}\right) \pi \lambda_{a} R_{2}^{2}}}{\left(1-p_{A} p_{U}\right)^{2}}-\frac{\pi \lambda_{a} R_{2}^{2}}{1-p_{A} p_{U}} e^{-\left(1-p_{A} p_{U}\right) \pi \lambda_{a} R_{2}^{2}}\right) .
\end{aligned}
$$

\section{Network Throughput}

The network throughput is defined as the sum of all users' achievable rates per unit area

$$
\mathcal{R}=\frac{\sum_{i=1}^{N} B \log _{2}\left(1+\operatorname{SINR}_{i}\right)}{\text { Area of network }},
$$

where $N$ refers to the number of APs in the network, and $\mathrm{SINR}_{i}$ denotes the SINR of the $i^{\text {th }} \mathrm{UE}$ with the $i^{\text {th }}$ AP. In this section, we derive the average network throughput,

$$
\begin{aligned}
\mathbb{E}[\mathcal{R}] & =\mathbb{E}\left[\frac{\sum_{i=1}^{N} B \log _{2}\left(1+\operatorname{SINR}_{i}\right)}{\text { Area of network }}\right] \\
& =\lambda_{a} B \mathbb{E}\left[\log _{2}(1+\operatorname{SINR})\right] \\
& \stackrel{(a)}{=} \frac{\lambda_{a} B}{\ln 2} \int_{0}^{\infty} \frac{P_{c}(T)}{1+t} d t,
\end{aligned}
$$

which can be viewed as the product of the AP density, total bandwidth and the average spectral efficiency, and 26(a) follows from the derivation in [16].

\section{NUMERICAL STUdY AND VALIDATION}

In this section, we elaborate numerical analysis on interference power, coverage probability, optimal AP density and average network throughput. Simulation results with Monte-Carlo methods are provided to validate the numerical evaluations of the derived models. The key parameters include frequency at $0.3 \mathrm{THz}$, bandwidth over $30 \mathrm{GHz}$, maximum transmit power of $15 \mathrm{dBm}$, and $\mu_{R}$ of -5 or $-15 \mathrm{~dB}$. The antenna beamwidths of APs and UEs are $\theta_{a}=\pi / 6, \varphi_{a}=\pi / 6, \theta_{u}=\pi / 3, \varphi_{u}=\pi / 3$. The densities of APs, wall blockers, and human blockers are set as $\lambda_{a}=0.1 \mathrm{~m}^{-2}, \lambda_{W}=0.1 \mathrm{~m}^{-1}, \lambda_{b}=0.3 \mathrm{~m}^{-2}$, respectively. The heights of APs, UEs and human bodies are $3 \mathrm{~m}, 1 \mathrm{~m}$ and $1.7 \mathrm{~m}$. The radius of the human-body is $0.3 \mathrm{~m}$.

\section{A. Mean Interference Power}

The mean interference power is calculated according to (15) and the mean value of the simulation data is evaluated by $\mathbb{E}\left[I_{\text {sim }}\right]$. The numerical studies for mean interference power with the nearest-AP user association are given in Fig. 10(a), Fig. 10(b) and Fig. 10(c), which show a good agreement between the analysis and the simulation for the mean interference power. As shown in Fig. 10(a), the interference power increases as the AP density becomes larger, which validates the deduction from (15) that the mean interference power is approximately proportional to $\lambda_{a}^{2}$. Since we use the unit $\mathrm{dB}$ for power, the curve in Fig. 10(a) is like a logarithmic function and gradually becomes saturate. Fig. 10(b) shows when there are more human blockers in the Tera-WLANs, the mean aggregated interference power at the tagged UE becomes weaker, because of the fact that the probability of interfering signals being blocked by the human body becomes higher. Also, from Fig. 10(c) we learn that the expected value of interference power gets higher when the wall blocker density becomes smaller, due to the increase of the number of the possible interfering APs, which makes the upper limit of the integral in (15) larger. For a typical scenario, $\lambda_{a}=0.1$, $\lambda_{b}=0.3, \lambda_{W}=0.1$, the aggregated mean interference power is approximately $-95 \mathrm{~dB}$. In addition, when the AP density is below 0.04 , or the wall density is less than 0.45 , the mean interference power will not exceed $-100 \mathrm{~dB}$, which is the noise power in our simulation. However, this can not be realized by increasing the human density.

\section{B. Coverage Probability}

The coverage probabilities are calculated using (18) for the nearest-AP user association and according to (21) for the LoSAP user association, respectively. The comparison between these two association schemes is given in Fig. 11(a). For the range of SINR threshold between 5 and $15 \mathrm{~dB}$, the coverage probability of LoS-AP user association scheme is $3 \%$ higher than the nearest-AP user association. In particular, at SINR threshold $=10 \mathrm{~dB}$, coverage probability is enhanced by over $6 \%$ owing to the proposed LoS-AP user association scheme. The simulation data is also leveraged to validate the accuracy of approximating the coverage probability.

The reason of the above results is explained as follows. On one hand, the reflection loss in the $\mathrm{THz}$ band is stronger than that in the microwave band and the received signal power averaged over fading from a farther LoS AP can be larger than that from a closer NLoS AP. From the proposed statistical THz channel model, one can suggest the AP-UE distance is not the best criterion for AP association in the THz network. Instead, a more appropriate strategy is to consider the LoS APs and then choose the nearest one from them. On the other hand, with the decrease of the coverage area of the $\mathrm{THz}$ network and more mobile devices are connected in the future, the density of the THz APs becomes larger, which leads to the high probability that the user can have at least one LoS AP.

In Fig. 11(b), we analyze the environment where the reflection loss is substantially severe, e.g., $\mu_{R}=-15 \mathrm{~dB}$. In this case, the signal power of NLoS rays is substantially lower than the LoS ray, which leads that the NLoS transmission can be neglected. When the SINR threshold is larger than $3 \mathrm{~dB}$, the theoretical results using the semi-closed-form expressions are fairly close to the simulated curves. Moreover, by comparing Fig. 11(a) and Fig. 11(b), it is shown that the coverage 


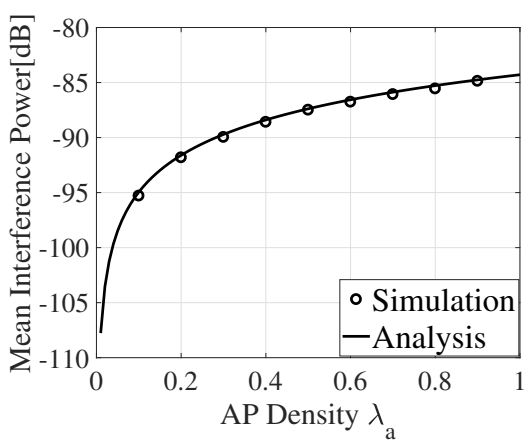

(a) Mean interference power versus the AP density.

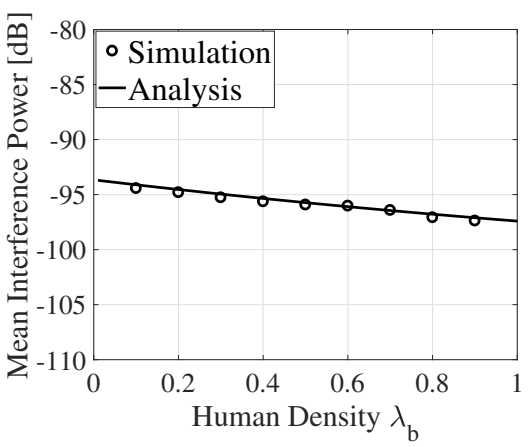

density.

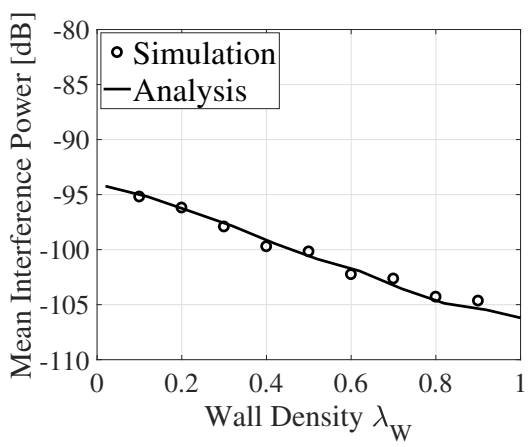

(c) Mean interference power versus the wall density.

Fig. 10. Mean interference power in the indoor Tera-WLANs is affected by the densities of the AP, the human and the wall.

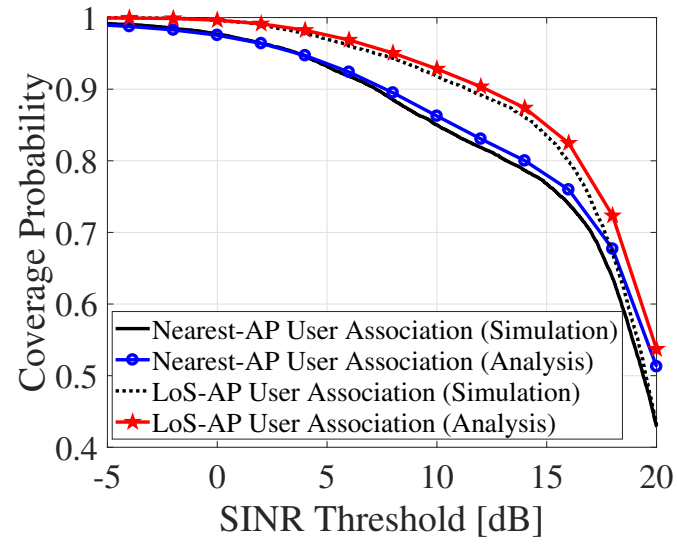

(a) Weak reflection loss, $\mu_{R}=-5 \mathrm{~dB}$.

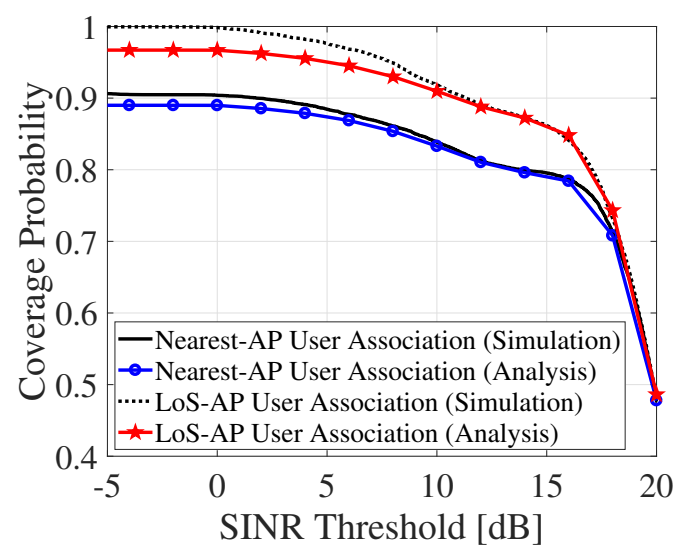

(b) Strong reflection loss, $\mu_{R}=-15 \mathrm{~dB}$.

Fig. 11. We validate the accuracy of the analytical expressions for the coverage probability by the simulations and compare two user association schemes. There is a good agreement between the analysis and the simulation.

probability is reduced in the environment with higher reflection loss when using the nearest-AP user association scheme, since the signal of NLoS APs become weaker.

In addition, we add comparisons between the proposed user association schemes, the random-AP user association scheme, and the max-SINR user association scheme [38]. In Fig. 12, we evaluate the coverage probability of the four association

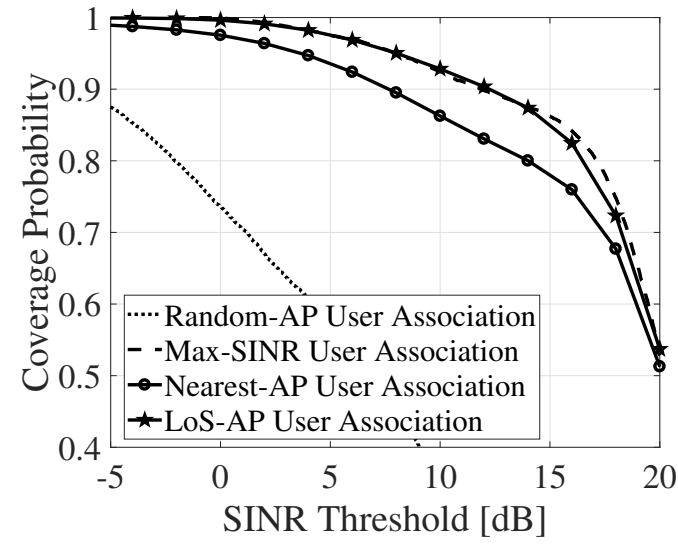

Fig. 12. Comparison of coverage probability between four user association schemes.

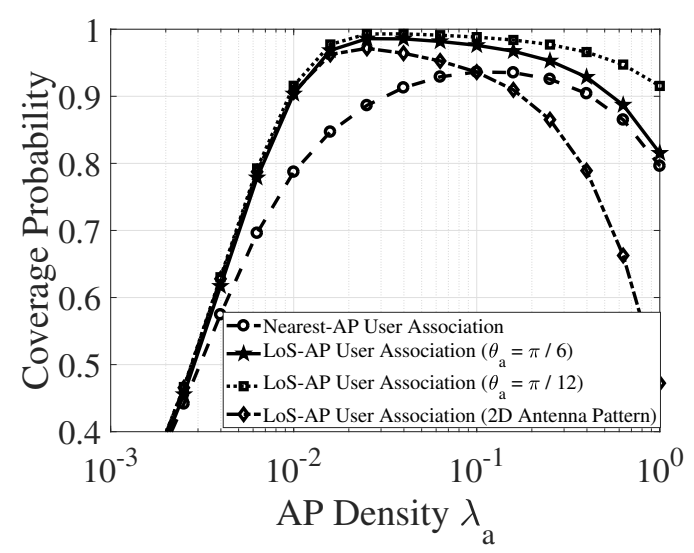

Fig. 13. Coverage probability versus the AP density for SINR threshold $T$ $=5 \mathrm{~dB}$.

schemes. The max-SINR user association is theoretically the best scheme to achieve the highest coverage probability, but may require frequent hand-off between different APs in practical networks. Among the other schemes, the performance of the proposed LoS-AP user association is close to the maxSINR association and substantially better than the nearest-AP association, which is evaluated as a benchmark. 


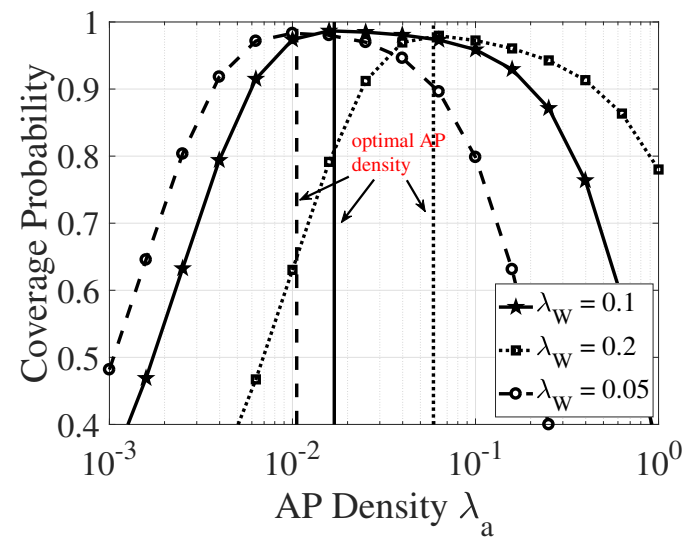

Fig. 14. The optimal AP density versus the wall density.

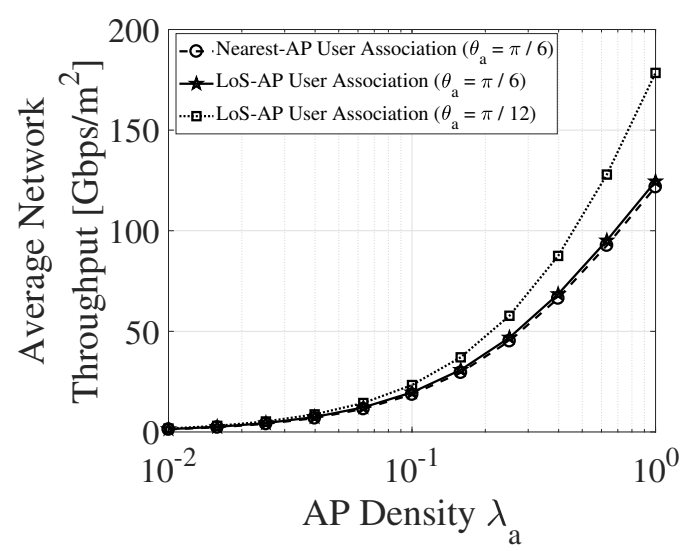

Fig. 15. Average network throughput versus the AP density.

\section{Optimal AP Density}

Next, by fixing the SINR threshold $T$ at $5 \mathrm{~dB}$, which is nearly the lowest requirement for reliable communications, we evaluate the coverage probability over the AP density. In Fig. 13, with the nearest-AP user association scheme, the coverage probability first improves while then reduces with the increase of the AP density. The optimal density appears at $0.15 \mathrm{~m}^{-2}$, at which the coverage probability can achieve 93\%. Analyzing the theoretical expressions in (18), (19) and (20), both the interference power and the received signal is increased by enlarging the AP density. When the AP density is less than the optimal solution, the improvement of the received signal power is stronger than that of the interference power. Above the optimal density, the network becomes much more denser and the coverage probability is reduced, caused by the enhancement of the interference power. At this moment, adding more APs with the density unchanged can increase the coverage area, but with more APs in the same-sized area the coverage probability is not able to be improved. By contrast, the optimal density of the LoS-AP association is about 0.04 $\mathrm{m}^{-2}$, which is smaller than that of the nearest-AP association.

In addition, when the planar beamwidth of the AP antenna pattern $\theta_{a}$ varies from $\pi / 6$ to $\pi / 12$, the transmitting antenna gain $G_{a}$ is increased by about $3 \mathrm{~dB}$, and the coverage is en- hanced by over $2 \%$ with the LoS-AP association for $\lambda_{a}>0.2$. Moreover, we compare the $3 \mathrm{D}$ pyramidal model with a $2 \mathrm{D}$ cone-shaped antenna model [17] and learn that the coverage probability is improved by over 3\% for $\lambda_{a}>0.05$ from using the $2 \mathrm{D}$ antenna pattern to the $3 \mathrm{D}$ pattern, essentially narrowing the vertical beam-width. It is shown that with narrower beamwidth of antenna pattern, the coverage probability can be effectively improved by compensating the path loss of the received signal power at the user.

In Fig. 14, we show the optimized design of the AP density for different $\lambda_{W}$. The vertical lines represent the optimal AP density that are calculated by using the closed-form expression (23) and solving the optimization problem (24). As the wall density increases, the value of the optimal AP density becomes larger. With larger $\lambda_{W}$, the average size of one interference region becomes smaller and larger AP density is required to maintain the average number of APs in the region and achieve the highest coverage probability.

\section{Network Throughput}

We evaluate the average network throughput versus the AP density in Fig. 15. From (26) we learn that the average network throughput is the product of the AP density and the integration of the coverage. The impact on the latter is not as much as that on the former when adding more APs. Although the coverage probability is reduced with more APs deployed in the network, the average network throughput improves. Numerically, the average network throughput increases from $20 \mathrm{Gbps}$ per $\mathrm{m}^{2}$ to $100 \mathrm{Gbps}$ per $\mathrm{m}^{2}$ when the AP density varies from 0.1 $\mathrm{m}^{-2}$ to $0.6 \mathrm{~m}^{-2}$. In addition, numerical results suggest that the LoS-AP user association can improve the network throughput by 2 Gbps per $\mathrm{m}^{2}$ when the AP density is larger than 0.4 $\mathrm{m}^{-2}$, compared to the nearest-AP user association. Moreover, when we strengthen the antenna gain by narrowing down the antenna beamwidth, the average network throughput is effectively improved by more than 20 Gbps-per $\mathrm{m}^{2}$ at the AP density over $0.4 \mathrm{~m}^{-2}$.

\section{CONCLUSION}

In this paper, we propose a new framework for downlink Tera-WLAN analysis. The wall blockage and human-body blockage in the indoor environment are considered. Based on analyzing the blockage effects, we develop a statistical $\mathrm{THz}$ channel model, equivalent to the multi-ray model. Then we present the interference analysis in terms of two user association schemes. With the moment generating functions of the aggregated interference, we gain the approximated coverage probability and the average network throughput. We present a closed-form expression of the coverage probability to calculate the optimal AP density.

With the numerical results, the proposed statistical channel model gives a good match to the multi-ray model. We can learn that the densification of APs and the sparsity of indoor blockage can increase the mean interference power. The coverage probability can be calculated with our approximated approach based on the MGF of the interference. For the nearest-AP user association scheme, the optimal AP density to maximize the 
coverage probability at $5 \mathrm{~dB}$ of SINR threshold is nearly 0.15 $\mathrm{m}^{-2}$. In this case, the coverage probability is about $93 \%$ and the average network throughput is approximately $30 \mathrm{Gbps} / \mathrm{m}^{2}$. By contrast, the LoS-AP user association can improve the coverage probability by 3 percent and the average network throughput by $2 \mathrm{Gbps} / \mathrm{m}^{2}$.

\section{APPENDIX A}

First we give the following lemma. Suppose that $Y_{i}=$ $X_{i} e^{j \theta_{i}}(i=1,2, \ldots, n)$ are independent complex random variables, the amplitudes $X_{i}$ are independent positive random variables, and the phases $\theta_{i}$ are independently and uniformly distributed in $[0,2 \pi)$, and $X_{i}, \theta_{i}$ are independent. Then the mean value of the modulus square, $\mathbb{E}\left[\left|\sum_{i=1}^{n} Y_{i}\right|^{2}\right]$, is given by $\mathbb{E}\left[\left|\sum_{i=1}^{n} Y_{i}\right|^{2}\right]=$ $\sum_{i=1}^{n} \mathbb{E}\left[X_{i}^{2}\right]$, and the mean value of the fourth power of the modulus, $\mathbb{E}\left[\left|\sum_{i=1}^{n} Y_{i}\right|^{4}\right]$ is derived as $\mathbb{E}\left[\left|\sum_{i=1}^{n} Y_{i}\right|^{4}\right]=$ $\sum_{i=1}^{n} \mathbb{E}\left[X_{i}^{4}\right]+\sum_{1 \leq i<j \leq n} 4 \mathbb{E}\left[X_{i}^{2}\right] \mathbb{E}\left[X_{j}^{2}\right]$.

Proof: The modulus square of the sum of the complex random variables is

$$
\left|\sum_{i=1}^{n} Y_{i}\right|^{2}=\sum_{i=1}^{n} X_{i}^{2}+\sum_{1 \leq i<j \leq n} 2 X_{i} X_{j} \cos \left(\theta_{i}-\theta_{j}\right) .
$$

Taking the expectations of both sides, we obtain

$$
\begin{aligned}
& \mathbb{E}\left[\left|\sum_{i=1}^{n} Y_{i}\right|^{2}\right] \\
& =\sum_{i=1}^{n} \mathbb{E}\left[X_{i}^{2}\right]+\sum_{1 \leq i<j \leq n} 2 \mathbb{E}\left[X_{i}\right] \mathbb{E}\left[X_{j}\right] \mathbb{E}\left[\cos \left(\theta_{i}-\theta_{j}\right)\right] \\
& \stackrel{(a)}{=} \sum_{i=1}^{n} \mathbb{E}\left[X_{i}^{2}\right]
\end{aligned}
$$

where 28(a) follows from $\mathbb{E}\left[\cos \left(\theta_{i}-\theta_{j}\right)\right]=$ $\int_{0}^{2 \pi} \int_{0}^{2 \pi} \cos \left(\theta_{i}-\theta_{j}\right) \frac{1}{2 \pi} d \theta_{i} \frac{1}{2 \pi} d \theta_{j}=0$. Following the similar way, we can derive $\mathbb{E}\left[\left|\sum_{i=1}^{n} Y_{i}\right|^{4}\right]$.

Proof of Lemma 3: In the absence of LoS path, the channel response of multi-ray model is

$$
H\left(u_{0}\right)=\sum_{i=1}^{N_{R}} H_{\mathrm{NLOS}}\left(u_{i}\right) e^{-j 2 \pi f \tau_{i}} .
$$

Because of the very short wavelength of the $\mathrm{THz}$ frequencies, even small movement can rotate the phase a lot and thus the phase of the each NLoS ray is indeed random. From the above lemma, we obtain $\mathbb{E}\left[\left|H\left(u_{0}\right)\right|^{2}\right]=N_{R} \mathbb{E}\left[\left|H_{\mathrm{NLoS}}\left(u_{i}\right)\right|^{2}\right]$. We do not account for the possibility that the NLoS ray is obstructed by the human body and take $N_{R}=4$. Fig. 5 shows the geometry relationship between $u_{0}$ and $u_{i}$, i.e., $u_{i}^{2}=u_{0}^{2}+4 t^{2}+4 t u_{0}|\cos \theta|$ for $i=1,2$ and $\cos \theta$ is replaced by $\sin \theta$ for $i=3,4$, where $t$ is an exponential random variable with the rate parameter $\lambda_{W}$ that denotes the $2 \mathrm{D}$ distance between the tagged UE and the surrounding wall. We neglect the little influence of the azimuth angle. Thus, the mean path loss of NLoS rays is

$$
\begin{aligned}
& \mathbb{E}\left[\left|H_{\mathrm{NLoS}}\left(u_{i}\right)\right|^{2}\right] \\
& =\left(\frac{c}{4 \pi f}\right)^{2} \mathbb{E}\left[R_{c}^{2}\right] \int_{0}^{\infty} \frac{\lambda_{W} e^{-\lambda_{W} t-\kappa \sqrt{u_{0}^{2}+4 t^{2}+\left(h_{a}-h_{u}\right)^{2}}}}{u_{0}^{2}+4 t^{2}+\left(h_{a}-h_{u}\right)^{2}} d t \\
& \stackrel{(a)}{\approx}\left(\frac{c}{4 \pi f}\right)^{2} \mathbb{E}\left[R_{c}^{2}\right] \int_{0}^{\infty} \frac{\lambda_{W} e^{-\lambda_{W} t}}{u_{0}^{2}+4 t^{2}+\left(h_{a}-h_{u}\right)^{2}} d t \\
& \stackrel{(b)}{\approx} \frac{c^{2} \mathbb{E}\left[R_{c}^{2}\right]}{4 \pi^{2} f^{2}\left(u_{0}^{2}+\left(h_{a}-h_{u}\right)^{2}\right)} \\
& \cdot \frac{1}{\left(1+\ln \left(1+\frac{4}{\left(u_{0}^{2}+\left(h_{a}-h_{u}\right)^{2}\right) \lambda_{W}^{2}}\right)\right)},
\end{aligned}
$$

where 30(a) follows the approximation that the absorption coefficient is small and 30(b) uses the approximation $\int_{0}^{\infty} \frac{e^{-x}}{x^{2}+a^{2}} d x=\int_{0}^{\infty} \frac{e^{-x}}{a^{2} e^{\ln \left(1+\frac{1}{a^{2}}\right) x}} d x=\frac{1}{a^{2}\left(1+\ln \left(1+\frac{1}{a^{2}}\right)\right)}$.

In the similar way, by employing the above lemma we are able to prove Lemma 4 .

\section{REFERENCES}

[1] Y. Wu et al., "Interference and Coverage Analysis for Indoor Terahertz Wireless Local Area Networks," in Proc. of IEEE Globecom Workshops, December 2019.

[2] A.-A. A. Boulogeorgos et al., "Terahertz technologies to deliver optical network quality of experience in wireless systems beyond $5 \mathrm{~g}$," IEEE Commun. Mag., vol. 56, no. 6, pp. 144-151, 2018.

[3] F. C. Commission, "Fcc takes steps to open spectrum horizons for new services and technologies." [Online]. Available: https: //docs.fcc.gov/public/attachments/DOC-356588A1.pdf

[4] Z. Zhang et al., "6g wireless networks: Vision, requirements, architecture, and key technologies," IEEE Veh. Technol. Mag., vol. 14, no. 3, pp. 28-41, 2019.

[5] I. F. Akyildiz et al., "Terahertz band: Next frontier for wireless communications," Physical Communication, vol. 12, pp. 16-32, 2014.

[6] C. Han et al., "Propagation modeling for wireless communications in the terahertz band," IEEE Commun. Mag., vol. 56, no. 6, pp. 96-101, 2018.

[7] J. M. Jornet et al., "Channel modeling and capacity analysis for electromagnetic wireless nanonetworks in the terahertz band," IEEE Trans. Wireless Commun., vol. 10, no. 10, pp. 3211-3221, October 2011.

[8] C. Han et al., "Multi-ray channel modeling and wideband characterization for wireless communications in the terahertz band," IEEE Trans. Wireless Commun., vol. 14, no. 5, pp. 2402-2412, 2014.

[9] J. Kokkoniemi et al., "Stochastic geometry analysis for mean interference power and outage probability in thz networks," IEEE Trans. Wireless Commun., vol. 16, no. 5, pp. 3017-3028, May 2017.

[10] N. Khalid et al., "Statistical characterization and analysis of low-thz communication channel for $5 \mathrm{~g}$ internet of things," Nano Commun. Networks, vol. 22, p. 100258, 2019.

[11] X.-W. Yao et al., "Stochastic geometry analysis of interference and coverage in terahertz networks," Nano Commun. Networks, vol. 13, pp. 9-19, 2017.

[12] M. Haenggi et al., "Stochastic geometry and random graphs for the analysis and design of wireless networks," IEEE J. Sel. Areas Commun., vol. 27, no. 7, pp. 1029-1046, Sep. 2009.

[13] R. W. Heath et al., "Modeling heterogeneous network interference using poisson point processes," IEEE Trans. Signal Process., vol. 61, no. 16, pp. 4114-4126, Aug 2013.

[14] H. ElSawy et al., "Stochastic geometry for modeling, analysis, and design of multi-tier and cognitive cellular wireless networks: A survey," IEEE Commun. Surveys Tuts., vol. 15, no. 3, pp. 996-1019, 2013.

[15] J. G. Andrews et al., "A tractable approach to coverage and rate in cellular networks," IEEE Trans. Commun., vol. 59, no. 11, pp. 31223134, November 2011. 
[16] T. Bai et al., "Coverage and rate analysis for millimeter-wave cellular networks," IEEE Trans. Wireless Commun., vol. 14, no. 2, pp. 11001114, Feb 2015.

[17] V. Petrov et al., "Interference and sinr in millimeter wave and terahertz communication systems with blocking and directional antennas," IEEE Trans. Wireless Commun., vol. 16, no. 3, pp. 1791-1808, March 2017.

[18] X. Yu et al., "Coverage analysis for millimeter wave networks: The impact of directional antenna arrays," IEEE J. Sel. Areas Commun., vol. 35, no. 7, pp. 1498-1512, 2017.

[19] R. Kovalchukov et al., "Evaluating sir in 3d millimeter-wave deployments: Direct modeling and feasible approximations," IEEE Trans. Wireless Commun., vol. 18, no. 2, pp. 879-896, Feb 2019.

[20] M. Rebato et al., "Stochastic geometric coverage analysis in mmwave cellular networks with realistic channel and antenna radiation models," IEEE Trans. Commun., vol. 67, no. 5, pp. 3736-3752, May 2019.

[21] Y. Zhou et al., "Coverage and rate analysis of millimeter wave noma networks with beam misalignment," IEEE Trans. Wireless Commun., vol. 17 , no. 12, pp. 8211-8227, 2018.

[22] M. Cheng et al., "Coverage analysis for millimeter wave cellular networks with imperfect beam alignment," IEEE Trans. Veh. Technol., vol. 67 , no. 9, pp. 8302-8314, 2018.

[23] M. Comisso et al., "Coverage analysis for $2 \mathrm{~d} / 3 \mathrm{~d}$ millimeter wave peerto-peer networks," IEEE Trans. Wireless Commun., vol. 18, no. 7, pp. $3613-3627,2019$.

[24] K. Venugopal et al., "Millimeter wave networked wearables in dense indoor environments," IEEE Access, vol. 4, pp. 1205-1221, 2016.

[25] M. Gapeyenko et al., "Analysis of human-body blockage in urban millimeter-wave cellular communications," in Proc. of IEEE ICC, May 2016.

[26] D. Maamari et al., "Coverage in mmwave cellular networks with base station co-operation," IEEE Trans. Wireless Commun., vol. 15, no. 4, pp. 2981-2994, 2016

[27] S. Biswas et al., "On the performance of relay aided millimeter wave networks," IEEE J. Sel. Topics Signal Process., vol. 10, no. 3, pp. 576588, April 2016

[28] T. Kürner et al., "Towards thz communications-status in research, standardization and regulation," Journal of Infrared, Millimeter, and Terahertz Waves, vol. 35, no. 1, pp. 53-62, 2014.

[29] D. He et al., "Stochastic channel modeling for kiosk applications in the terahertz band," IEEE Trans. THz Sci. Technol., vol. 7, no. 5, pp. 502-513, Sep. 2017.

[30] S. Kim et al., "Statistical modeling and simulation of short-range deviceto-device communication channels at sub-thz frequencies," IEEE Trans. Wireless Commun., vol. 15, no. 9, pp. 6423-6433, Sep. 2016.

[31] C. Han et al., "On medium access control schemes for wireless networks in the millimeter-wave and Terahertz bands," Nano Commun. Networks, vol. 19, pp. 67-80, 2019.

[32] M. K. Müller et al., "Analyzing wireless indoor communications by blockage models," IEEE Access, vol. 5, pp. 2172-2186, 2017.

[33] T. Bai et al., "Analyzing uplink sinr and rate in massive mimo systems using stochastic geometry," IEEE Trans. Commun., vol. 64, no. 11, pp. 4592-4606, 2016

[34] B. A. Bilgin et al., "Human blockage model for indoor terahertz band communication," in Proc. of IEEE ICC Workshops, 2019.

[35] A. Moldovan et al., "Coverage and achievable rate analysis for indoor terahertz wireless networks," in Proc. of IEEE ICC, 2017.

[36] A. Shafie et al., "Coverage analysis for 3d terahertz communication systems with blockage and directional antennas," in Proc. of IEEE ICC Workshops, 2020.

[37] H. Alzer, "On some inequalities for the incomplete gamma function," Mathematics of Computation, vol. 66, no. 218, pp. 771-778, 1997.

[38] A. Alizadeh et al., "A study of interference distributions in millimeter wave cellular networks," in Proc. of IEEE COMCAS, 2019.

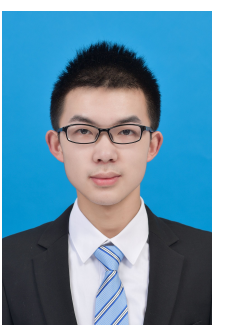

Yongzhi Wu (S'19) received B.E degree in Electronic and Information Engineering from Huazhong University of Science and Technology in 2019. Since 2019, he is pursuing Ph.D. degree in the Terahertz Wireless Communication Laboratory, Shanghai Jiao Tong University. His research interests include Terahertz communications, joint communications and sensing.

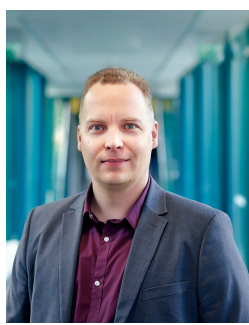

Joonas Kokkoniemi (S'12-M'18) received his B.Sc (Tech.), M.Sc. (Tech.), and Dr.Sc. (Tech.) degrees in communications engineering from the University of Oulu, Finland, in 2011, 2012, and 2017, respectively. $\mathrm{He}$ is a postdoctoral researcher with the Centre for Wireless Communications, University of Oulu. From September to November 2013, he was a visiting researcher at Tokyo University of Agriculture and Technology, Japan. From March to October 2017 , he was a visiting postdoctoral researcher with the University at Buffalo, New York. His research interests include $\mathrm{THz}$ band channel and system modeling.

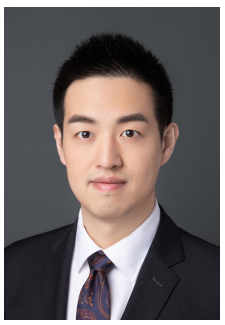

Chong Han (M'16) received Ph.D. degree in Electrical and Computer Engineering from Georgia Institute of Technology, USA in 2016. He is currently a tenure-track Associate Professor with the Terahertz Wireless Communications (TWC) Laboratory, University of Michigan-Shanghai Jiao Tong University (UM-SJTU) Joint Institute, Shanghai Jiao Tong University, China. He is the recipient of 2018 Elsevier NanoComNet (Nano Communication Network Journal) Young Investigator Award, 2017 Shanghai Sailing Program 2017, and 2018 Shanghai ChenGuang Program. He is an editor with IEEE Open Journal of Vehicular Technology since 2020, an associate editor with IEEE Access since 2017, an editor with Elsevier Nano Communication Network journal since 2016, and is a TPC chair to organize multiple IEEE and ACM conferences and workshops. His research interests include Terahertz band and millimeter-wave communication networks, and electromagnetic nanonetworks. He is a member of the IEEE and ACM.

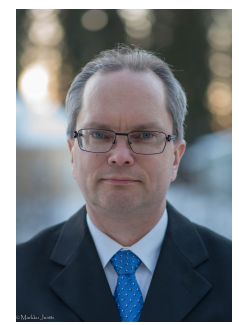

Markku Juntti (S'93-M'98-SM'04-F'20) received his M.Sc. (EE) and Dr.Sc. (EE) degrees from University of Oulu, Oulu, Finland in 1993 and 1997, respectively.

Dr. Juntti was with University of Oulu in 1992-98. In academic year 1994-95, he was a Visiting Scholar at Rice University, Houston, Texas. In 1999-2000, he was a Senior Specialist with Nokia Networks. Dr. Juntti has been a professor of communications engineering since 2000 at University of Oulu, Centre for Wireless Communications (CWC), where he leads the Communications Signal Processing (CSP) Research Group. He also serves as Head of CWC - Radio Technologies (RT) Research Unit. His research interests include signal processing for wireless networks as well as communication and information theory. $\mathrm{He}$ is an author or co-author in almost 500 papers published in international journals and conference records as well as in books Wideband CDMA for UMTS in 2000-2010, Handbook of Signal Processing Systems in 2013 and 2018 and 5G Wireless Technologies in 2017. Dr. Juntti is also an Adjunct Professor at Department of Electrical and Computer Engineering, Rice University, Houston, Texas, USA.

Dr. Juntti was an Editor of IEEE TRANSACTIONS ON COMMUNICATIONS and an Associate Editor for IEEE TRANSACTIONS ON VEHICULAR Technology. He was Secretary of IEEE Communication Society Finland Chapter in 1996-97 and the Chairman for years 2000-01. He has been Secretary of the Technical Program Committee (TPC) of the 2001 IEEE International Conference on Communications (ICC), and the Chair or CoChair of the Technical Program Committee of several conferences including 2006 and 2021 IEEE International Symposium on Personal, Indoor and Mobile Radio Communications (PIMRC), the Signal Processing for Communications Symposium of IEEE Globecom 2014, Symposium on Transceivers and Signal Processing for 5G Wireless and mm-Wave Systems of IEEE GlobalSIP 2016, ACM NanoCom 2018, and 2019 International Symposium on Wireless Communication Systems (ISWCS). He has also served as the General Chair of 2011 IEEE Communication Theory Workshop (CTW 2011). 